S. Kozlova, A. Mialdun, I. Ryzhkov, T. Janzen, J. Vrabec, V. Shevtsova

\title{
Do ternary liquid mixtures exhibit negative main Fick diffusion coefficients?
}

Journal article | Accepted manuscript (Postprint)

This version is available at https://doi.org/10.14279/depositonce-8389

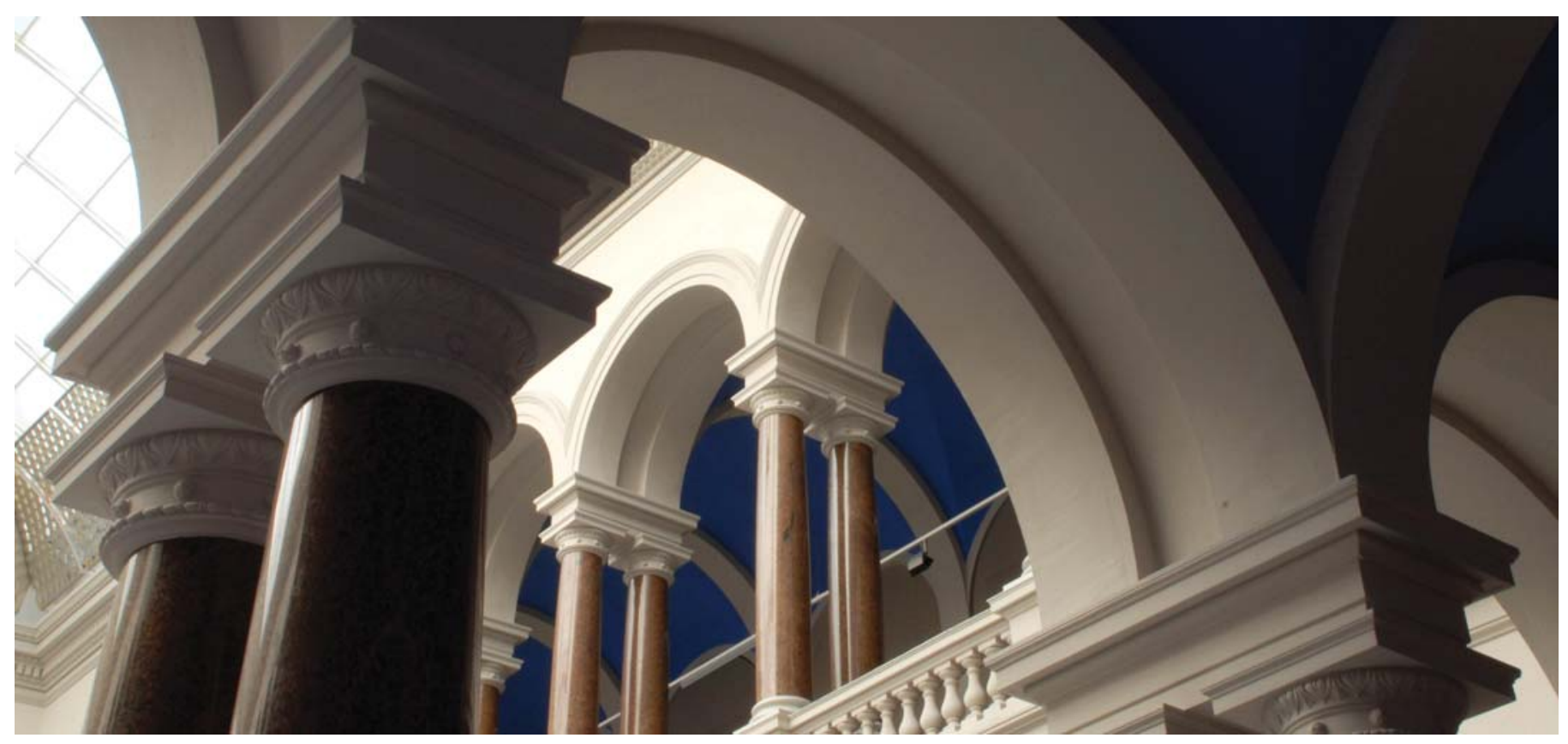

Kozlova, S.; Mialdun, Aliaksandr; Ryzhkov, I.; Janzen, Tatjana; Vrabec, Jadran; Shevtsova, Valentina (2019). Do ternary liquid mixtures exhibit negative main Fick diffusion coefficients? Physical Chemistry,

Chemical Physics, 21(4), 2140-2152. https://doi.org/10.1039/c8cp06795c 


\title{
Do ternary liquid mixtures exhibit negative main Fick diffusion coefficients?
}

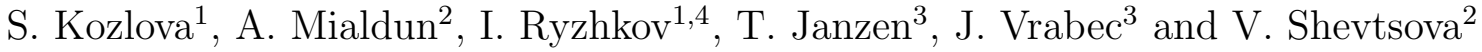 \\ ${ }^{1}$ Institute of Computational Modelling SB RAS, \\ 50/44 Akademgorodok, 660036, Krasnoyarsk, Russia \\ 2 Microgravity Research Center, Université Libre de Bruxelles (ULB), \\ CP-165/62, Av. F.D. Roosevelt, 50, B-1050 Brussels, Belgium \\ Thermodynamics and Thermal Process Engineering, Technical University Berlin, \\ Ernst-Reuter-Platz 1, 10587 Berlin, Germany \\ ${ }^{4}$ Siberian Federal University \\ 79 Svobodny pr., 660041, Krasnoyarsk, Russia
}

Experimental data on Fick diffusion coefficients of ternary and higher mixtures depend on the reference frame; those which are in common use are associated with the average velocity either with respect to volume, mass or mole number. In this study, the dependence of diffusion coefficients on the reference frame is thoroughly analyzed for three ternary mixtures of different types. The first one, tetralin - isobutylbenzene - dodecane, can almost be considered as ideal, the second one, cyclohexane - toluene - methanol, exhibits liquid-liquid phase separation and the third one, water - ethanol - triethylene glycol, contains three associating species and is also strongly non-ideal. Experimental diffusion coefficient data sampled in the volume reference frame are transformed to the molar and mass reference frames. The required partial molar volumes are derived from present density measurements. Four additional mixtures are considered along a single or two compostion paths. A highlight of this study is the existence of a strong similarity of the main diffusion coefficients in the volume and mass reference frames for all considered mixtures. When the excess volume is small, the coefficients in the molar reference frame are also similar. However, for the mixture with a large excess volume (containing water), the diffusion coefficients in the molar reference frame differ significantly, even indicating negative main diffusion coefficients. It is shown that negative main diffusion coefficients appear due to relatively large experimental uncertainties of cross diffusion coefficients, which are propagated and amplified by frame transformation.

Keywords: Ternary mixture, Fick diffusion coefficients, molar volumes, reference frames, DCMIX.

\section{Introduction}

Diffusive mass transport in liquids is a fundamental process, which plays an important role in chemical engineering as well as in biological systems, and is often described by Fick's law. This approach relates a diffusive flux of a species to gradients of concentration, mole fraction or mass fraction [1]. Diffusion coefficients are defined as proportionality constants between a flux and a driving force gradient. Recently, the scientific community has taken ternary mixtures more into focus because they can be seen as prototypes for truly multicomponent mixtures. In ternary mixtures, the $(2 \times 2)$ Fick diffusion coefficient matrix is not symmetric in most cases. Cross diffusion coefficients $D_{i j}$, which characterize a flux of one species $i$ induced by the driving 
force gradient of another species $j$, can lead to spatial and spatiotemporal pattern formation, as well as instabilities [2,3]. Moreover, the numerical values of the Fick diffusion coefficients of ternary mixtures depend on the order of components and also on the reference frame, for which the diffusive fluxes are expressed [1]. Only the eigenvalues of the Fick diffusion matrix are independent on the order of components and the reference frame.

To determine Fick diffusion coefficients, experimental methods, molecular simulation, theoretical or empirical approaches are used. Regardless of the experimental technique, extracting four diffusion coefficients $D_{i j}$ from laboratory raw data is challenging because of several reasons. Most methods that are applicable to ternary mixtures, e.g. the Rayleigh [4] and Gouy [5] interferometric approaches, the Taylor dispersion technique [6-8], sliding symmetric tubes [9] or digital interferometry in the counter flow cell [10], are based on the optical properties of liquids [11], where a key role is played by the variation of the refractive index $n$ with concentration c. Ternary mixtures can be processed relatively easily when the two contrast factors $\partial n / \partial c_{i}$, where $i=1,2$, have similar orders of magnitude. However, for many mixtures of interest this is not the case, which significantly complicates experimental work. The accuracy of the cross diffusion coefficients is particularly sensitive to the contrast factor ratio $\left(\partial n / \partial c_{1}\right) /\left(\partial n / \partial c_{2}\right)$. Another difficulty arises from convergence problems of fitting procedures, which often occur in conjunction with the Taylor dispersion technique [12]. As a result, experimental Fick diffusion coefficient data are only available for a very limited number of ternary mixtures [13]. In this context, molecular dynamics simulation may provide valuable support when it is simultaneously applied to the same system. However, such studies are still rare [14-16].

Fitting by nonlinear regression, which minimizes the residue between experimental raw data and working equations, is usually implemented under the constraints $[1,17]$

$$
\begin{aligned}
D_{11}+D_{22} & \geq 0, \\
D_{11} D_{22}-D_{12} D_{21} & \geq 0, \\
\left(D_{11}-D_{22}\right)^{2}+4 D_{12} D_{21} & \geq 0 .
\end{aligned}
$$

These thermodynamic stability conditions [17] do not require the main diffusion coefficients to be individually positive

$$
D_{11} \geq 0, \quad D_{22} \geq 0 .
$$

Kirkaldy and Purdy [18] as well as Clark and Rowley [19] argued that very non-ideal solutions, near their plait point, may exhibit negative main Fick diffusion coefficients. However, the majority of experimental results, also those for mixtures with liquid-liquid phase separation $[7,8]$, suggest the general validity of requirement (4) [20,21]. Negative main Fick diffusion coefficients violate physical intuition because they indicate that species oppose their dilution even in the absence of cross diffusion effects. Today, the validity of requirements (3) and (4) is thus under discussion $[13,22]$. Note that the negative main diffusion coefficients reported in the literature usually appeared in the course of transformations between different reference frames or when changing the order of components $[5,13,23]$. As a rule, it is considered that experiments are performed in the volume reference frame. There are three common reference frames for expressing Fick's law: volume-, mass- or molar-averaged. There is therefore a variety of diffusion coefficients in use.

In preceding work of our group, $D_{i j}$ data of several ternary systems were measured $[12$, $24,25]$ and it was found that requirements (3) and (4) are both valid. The aim of the present study is to transform experimental diffusion coefficients from the volume reference frame to other reference frames and assess requirement (4). When the main diffusion coefficients $D_{i i}$ 
were reported to be negative, the original experimental data were carefully analyzed, in particular with respect to the uncertainties of the cross diffusion coefficients. For this purpose, three ternary mixtures were chosen, which were measured on the International Space Station during the DCMIX (Diffusion and thermodiffusion Coefficients in MIXtures) mission [26-29]. The first system is an almost ideal mixture, which is composed of tetralin (THN), isobutylbenzene (IBB) and dodecane $\left(\mathrm{nC}_{12}\right)[12,25]$. For that system, the transport coefficients of its binary subsystems are also well-known [30-32]. The second system, composed of cyclohexane, toluene and methanol, exhibits a miscibility gap $[27,28,33]$. The third system, composed of water, ethanol and triethylene glycol (TEG), is highly non-ideal [24] and contains a region with poor optical properties $[34,35]$. The DCMIX project focused on measurements of Soret, thermodiffusion and Fick diffusion coefficients. However, it was understood that from thermodiffusion experiments with two-wavelength interferometry, it is impossible to determine six unknowns, i.e. two Soret and four Fick diffusion coefficients. Hence, the diffusion coefficients had to be measured independently on the ground. Furthermore, the governing equations for thermodiffusion are written in the mass reference frame [36] which necessitates transformation of diffusion coefficients measured in the volume reference frame. Understanding this bridge is an essential motivation for the current study. In accordance with the DCMIX program, it will be referred to these mixtures as DCMIX1, DCMIX2 and DCMIX3.

\section{Diffusion matrices in different reference frames}

There are three common forms of the generalized Fick's law: (1) molar flux $\mathbf{J}^{V}$ with respect to volume-averaged velocity; (2) molar flux $\mathbf{J}^{M}$ with respect to molar-averaged velocity; (3) mass flux $\mathbf{J}^{m}$ with respect to mass-averaged velocity.

When the driving force is expressed in terms of the molar concentration gradient $\nabla c_{j}$, the diffusive molar flux of component $i$ is related to the volume-averaged velocity, leading to the volume reference frame

$$
J_{i}^{V}=c_{i}\left(u_{i}-u_{0}^{V}\right)=-\sum_{j=1}^{n-1} D_{i j}^{V} \nabla c_{j}, \quad \sum_{j=1}^{n} c_{j}=c_{t} .
$$

Therein, $c_{i}$ is the molar concentration of component $i, u_{0}^{V}=\sum_{i=1}^{n} \phi_{i} u_{i}$ the average volume velocity, $\phi_{i}$ the volume fraction, $u_{i}$ the velocity of component $i$ and $D_{i j}^{V}$ the Fick diffusion coefficients in that reference frame.

If the driving force is related to the gradient of mole fraction $\nabla x_{j}$, the corresponding diffusive molar flux is defined in the molar reference frame as

$$
J_{i}^{M}=c_{i}\left(u_{i}-u_{0}^{M}\right)=-c_{t} \sum_{j=1}^{n-1} D_{i j}^{M} \nabla x_{j}, \quad \sum_{j=1}^{n} x_{j}=1 .
$$

Therein, $u_{0}^{M}=\sum_{i=1}^{n} x_{i} u_{i}$ is the average molar velocity, $x_{i}$ the mole fraction and $D_{i j}^{M}$ the corresponding diffusion coefficients.

Alternatively, the mass flux can be expressed in terms of the driving force induced by the mass fraction gradient $\nabla w_{j}$, leading to the mass reference frame

$$
J_{i}^{m}=\rho_{i}\left(u_{i}-u_{0}^{m}\right)=-\rho \sum_{j=1}^{n-1} D_{i j}^{m} \nabla w_{j}, \quad \sum_{j=1}^{n} w_{j}=1 .
$$


Therein, $\rho$ is the specific mixture density, $\rho_{i}$ the mass concentration of component $i, u_{0}^{m}=$ $\sum_{i=1}^{n} w_{i} u_{i}$ the average mass velocity, $w_{i}$ the mass fraction and $D_{i j}^{m}$ the corresponding diffusion coefficients.

For a mixture containing $n$ components, Fick's approach involves $n-1$ independent diffusion fluxes and $(n-1) \times(n-1)$ diffusion coefficients. The main diffusion coefficients $D_{i i}$, standing for an arbitrary reference frame, connect the flux of component $i$ to its own driving force gradient. The cross diffusion coefficients $D_{i j}$ describe the flux of component $i$ induced by the driving force gradient of component $j$, which can be understood as a coupled action. Accordingly, the diffusion coefficient matrices $\boldsymbol{D}^{V}, \boldsymbol{D}^{M}$ and $\boldsymbol{D}^{m}$ in volume, molar and mass reference frames will, in general, not be the same.

Diffusion coefficient measurements for ternary mixtures were carried out during the DCMIX mission with the Taylor dispersion technique, which was originally assigned to the volume reference frame [1]. In this frame, the weighted sum of molar fluxes is

$$
\sum_{i=1}^{n} v_{i} J_{i}^{V}=0
$$

where $v_{i}$ is the partial molar volume of component $i$, which will be discussed in the next section.

For the molar and mass reference frames, the following closures hold

$$
\sum_{i=1}^{n} J_{i}^{M}=\sum_{i=1}^{n} J_{i}^{m}=0 .
$$

Of course, diffusive fluxes $\boldsymbol{J}^{V}, \boldsymbol{J}^{M}$ and $\boldsymbol{J}^{m}$ are related, which allows to express the diffusion coefficients in different reference frames. In this way, the diffusion coefficient matrix in the volume reference frame $\boldsymbol{D}^{V}$ obtained experimentally can be transformed into matrices in the molar and mass reference frames, $\boldsymbol{D}^{M}$ and $\boldsymbol{D}^{m}$, respectively.

The diffusion matrix in the volume reference frame $\boldsymbol{D}^{V}$ is related to the one in the molar reference frame $\boldsymbol{D}^{M}$ by [1]

$$
\boldsymbol{D}^{M}=\boldsymbol{B}^{M V} \boldsymbol{D}^{V} \boldsymbol{B}^{V M},
$$

where the elements of matrices $\boldsymbol{B}^{M V}$ and $\boldsymbol{B}^{V M}$ are

$$
B_{i k}^{V M}=\delta_{i k}-\frac{x_{i}}{v}\left(v_{k}-v_{n}\right), \quad B_{i k}^{M V}=\delta_{i k}-x_{i}\left(1-\frac{v_{k}}{v_{n}}\right), \quad i, k=1,2, n=3 .
$$

Therein, $\delta_{i k}$ is Kronecker delta, $v=M / \rho$ the molar volume of the mixture, $M$ its molar mass and $v_{i}$ the partial molar volumes.

When matrix $\boldsymbol{D}^{M}$ is known, the relation between the diffusion coefficient matrices in the mass and molar reference frames is given by [1]

$$
\boldsymbol{D}^{m}=\boldsymbol{B}^{m M}[w][x]^{-1} \boldsymbol{D}^{M}[x][w]^{-1} \boldsymbol{B}^{M m},
$$

where the elements of matrices $\boldsymbol{B}^{m M}$ and $\boldsymbol{B}^{M m}$ are

$$
B_{i k}^{M m}=\delta_{i k}-w_{i}\left(\frac{x_{k}}{w_{k}}-\frac{x_{n}}{w_{n}}\right), \quad B_{i k}^{m M}=\delta_{i k}-w_{i}\left(1-\frac{w_{n} x_{k}}{x_{n} w_{k}}\right), \quad i, k=1,2, n=3 .
$$

Therein, $[w]=\operatorname{diag}\left\{w_{i}\right\}$ and $[x]=\operatorname{diag}\left\{x_{i}\right\}$ are the diagonal matrices of mass and mole fractions, respectively. 

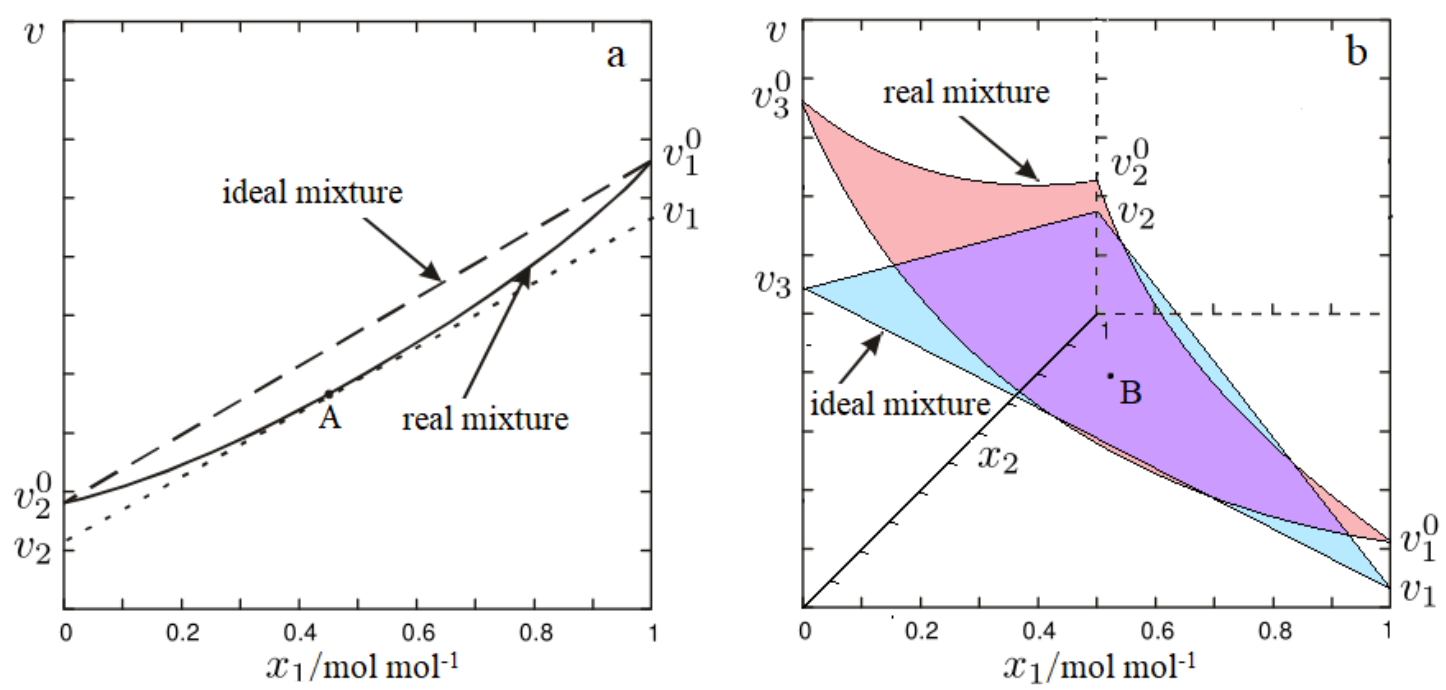

Figure 1: Molar volume of a mixture $v$ as a function of mole fraction(s), showing (a) the tangent line at composition A of a binary mixture or (b) the tangent plane at composition B of a ternary mixture.

\section{Partial molar volumes}

To convert the diffusion matrix between different reference frames, the partial molar volumes are crucial. They stand for the contribution of one substance to the volume of a mixture at constant temperature $T$ and pressure $p$ and are defined by $v_{i}=\left(\partial V / \partial n_{i}\right)_{T, p, n_{j}}$. They represent the change of the (large) volume of a mixture upon addition of a (small) molar amount of component $i$.

The molar volume of a binary liquid mixture at constant temperature and pressure is in general given by

$$
v=x_{1} v_{1}+x_{2} v_{2} .
$$

On the other hand, the molar volume of a volumetrically ideal binary mixture is

$$
v=\frac{M}{\rho}=\frac{x_{1} M_{1}}{\rho}+\frac{x_{2} M_{2}}{\rho}=x_{1} v_{1}^{0}+x_{2} v_{2}^{0},
$$

where $M_{i}$ stands for the molar mass of component $i$.

In a volumetrically non-ideal mixture, the total volume is not the weighted sum of the pure component volumes $v_{i}^{0}$ and the partial molar volumes $v_{i}$ depend on mixture composition such that they can be smaller or larger than $v_{i}^{0}$ due to volumetric contraction or dilatation. Working with Eq. (11) under isothermal-isobaric conditions and applying the Gibbs-Duhem equation leads to the following expression for the partial molar volume (details can be found in the supplementary material)

$$
v_{1}=v+\left(1-x_{1}\right) \frac{d v}{d x_{1}} .
$$

A schematic of the partial molar volumes is given in Fig. 1. If the mole fraction dependence of $v$ is known, the tangent line at the composition of interest (point A) has the slope $d v / d x_{1}$ in Eq. (13). This tangent line can be interpreted as the addition to the molar volume of a hypothetical ideal mixture with the components' molar volumes $v_{1}$ and $v_{2}$. Accordingly, the values of $v_{1}$ and $v_{2}$ obviously depend on mixture composition. 
For ternary mixtures, the mathematical basis is the same as in case of binary mixtures and the total molar volume can be expressed by

$$
v=\frac{M}{\rho}=\frac{x_{1} M_{1}+x_{2} M_{2}+\left(1-x_{1}-x_{2}\right) M_{3}}{\rho} .
$$

In analogy to a binary mixture, the partial molar volumes of a ternary mixture can be obtained by

$$
\begin{aligned}
v_{1} & =v+\left(1-x_{1}\right) \frac{\partial v}{\partial x_{1}}-x_{2} \frac{\partial v}{\partial x_{2}} \\
v_{2} & =v-x_{1} \frac{\partial v}{\partial x_{1}}+\left(1-x_{2}\right) \frac{\partial v}{\partial x_{2}} \\
v_{3} & =v-x_{1} \frac{\partial v}{\partial x_{1}}-x_{2} \frac{\partial v}{\partial x_{2}} .
\end{aligned}
$$

The calculation of partial molar volumes of ternary mixtures leads to a three-dimensional problem (Fig. 1b). The mixture molar volume $v$ is now a surface and the derivatives $\partial v / \partial x_{1}$ and $\partial v / \partial x_{2}$ in Eqs. (15) to (18) characterize a tangent plane for the composition of interest (point B in Fig. 1b).

In the following, density, partial molar and excess volumes of three ternary mixtures are analyzed, namely $\mathrm{THN}-\mathrm{IBB}-\mathrm{nC}_{12}$, cyclohexane - toluene - methanol and water - ethanol TEG. To determine these quantities, the density was measured in the present work. Analyzing the raw data, it was noted that the molar volume is an almost linear function of the mole fractions $x_{1}$ and $x_{2}$. To rationalize data processing, it was started by calculating molar volumes with Eq. (14) from discrete density measurements and then matching this data set with a second-order polynomial. From that correlation, density and excess volume were calculated over the full composition space, which provided small average absolute deviations (AAD) between measurements and polynomial.

\subsection{THN - IBB $-\mathbf{n C}_{12}($ DCMIX1)}

The density of the ternary mixture $\mathrm{THN}-\mathrm{IBB}-\mathrm{nC}_{12}$ was measured for 31 compositions with a DMA 5000 vibrating tube density meter of Anton Paar with an accuracy of $5 \cdot 10^{-6} \mathrm{~g} / \mathrm{cm}^{3}$ and a temperature repeatability of $0.001 \mathrm{~K}$. The molar volume of the ternary mixture was obtained from these density values by a second-order polynomial in terms of $x_{1}$ and $x_{2}$. For this mixture, the correlation for the molar volume takes the form

$$
v /\left(\mathrm{cm}^{3} / \mathrm{mol}\right)=\left(\begin{array}{lll}
1 & x_{1} & x_{1}^{2}
\end{array}\right)\left[\begin{array}{ccc}
228.52 & -69.30 & -1.11 \\
-91.29 & -1.90 & 0 \\
-0.24 & 0 & 0
\end{array}\right]\left(\begin{array}{c}
1 \\
x_{2} \\
x_{2}^{2}
\end{array}\right)=\left(x_{1}^{i}\right) \cdot\left[P_{i, j}\right] \cdot\left(x_{2}^{j}\right)^{T} .
$$

The three-dimensional surface of the molar volume is presented in Fig. 2b. Indeed, its flat shape confirms an almost ideal volumetric behavior of this mixture. The excess molar volume is presented in Fig. 2c, which, as expected, is negligibly small. The density is shown in Fig. 2a and the average absolute deviation between measurements and correlation is in this case only $0.03 \%$. 

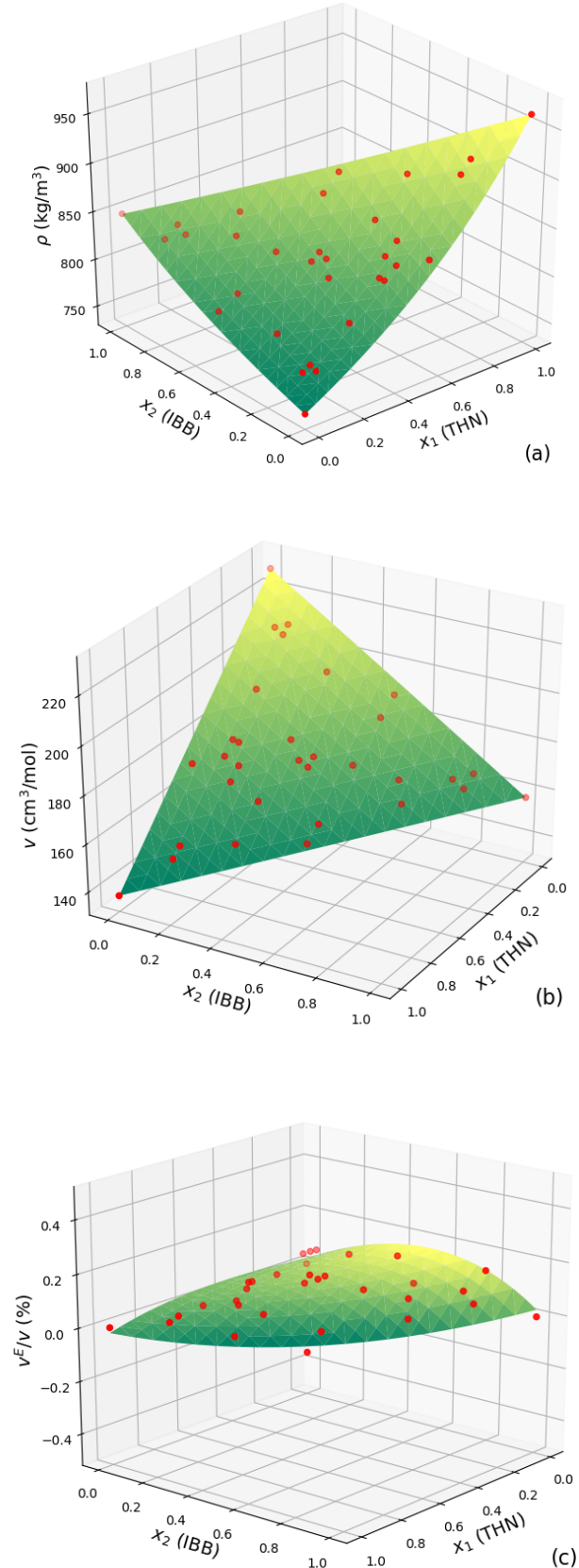

Figure 2: Ternary mixture $\mathrm{THN}(1)-\mathrm{IBB}(2)-\mathrm{nC}_{12}$ (3): (a) specific density $\rho$; (b) molar volume $v$; (c) relative molar excess volume $v^{\mathrm{E}} / v$. 
Consequently, the derivatives $\partial v / \partial x_{1}$ and $\partial v / \partial x_{2}$ are given by

$$
\begin{gathered}
\frac{\partial v}{\partial x_{1}}=\frac{\partial\left(x_{1}^{i}\right)}{\partial x_{1}} \cdot\left[P_{i, j}\right] \cdot\left(x_{2}^{j}\right)^{T}=\left(\begin{array}{lll}
0 & 1 & 2 x_{1}
\end{array}\right) \cdot\left[P_{i j}\right] \cdot\left(\begin{array}{c}
1 \\
x_{2} \\
x_{2}^{2}
\end{array}\right), \\
\frac{\partial v}{\partial x_{2}}=\left(\begin{array}{lll}
1 & x_{1} x_{1}^{2}
\end{array}\right) \cdot\left[P_{i, j}\right] \cdot\left(\frac{\partial\left(x_{2}^{j}\right)}{\partial x_{2}}\right)^{T}=\left(\begin{array}{lll}
1 & x_{1} & x_{1}^{2}
\end{array}\right) \cdot\left[P_{i j}\right] \cdot\left(\begin{array}{c}
0 \\
1 \\
2 x_{2}
\end{array}\right) .
\end{gathered}
$$

Using these derivatives, the partial molar volumes $v_{i}$ can be calculated with Eqs. (15) to (17).

\subsection{Cyclohexane - toluene - methanol (DCMIX2)}

The density of the ternary mixture cyclohexane - toluene - ethanol was measured at 65 compositions and the molar volumes were calculated with Eq. (14). Based on these data, the following correlation was found

$$
v /\left(\mathrm{cm}^{3} / \mathrm{mol}\right)=\left(\begin{array}{lll}
1 & x_{1} & x_{1}^{2}
\end{array}\right)\left[\begin{array}{ccc}
40.79 & 65.79 & 0.30 \\
70.79 & -0.18 & 0 \\
-2.83 & 0 & 0
\end{array}\right]\left(\begin{array}{c}
1 \\
x_{2} \\
x_{2}^{2}
\end{array}\right)=\left(x_{1}^{i}\right) \cdot\left[P_{i, j}\right] \cdot\left(x_{2}^{j}\right)^{T} .
$$

Three-dimensional surfaces of the density, molar volume and excess volume are shown in Fig. 3 as a function of mole fractions. Although this mixture contains a miscibility gap at $x_{2} \rightarrow 0 \quad[7,8]$, the molar volume exhibits an almost perfectly flat shape. The excess volume increases towards the miscibility gap, but its value does not exceed $1 \%$. The density surface has a more complex shape and emphasizes that it was appropriate to fit the molar volume, not the density. The average absolute deviation between measurements and correlation is in this case $0.07 \%$.

\subsection{Water - ethanol - TEG (DCMIX3)}

The density of the ternary mixture water - ethanol - TEG was measured at 67 compositions and the correlation for its molar volume is

$$
v /\left(\mathrm{cm}^{3} / \mathrm{mol}\right)=\left(\begin{array}{lll}
1 & x_{1} & x_{1}^{2}
\end{array}\right)\left[\begin{array}{ccc}
134.35 & -80.22 & 4.68 \\
-120.64 & 4.18 & 0 \\
4.29 & 0 & 0
\end{array}\right]\left(\begin{array}{c}
1 \\
x_{2} \\
x_{2}^{2}
\end{array}\right)=\left(x_{1}^{i}\right) \cdot\left[P_{i, j}\right] \cdot\left(x_{2}^{j}\right)^{T} .
$$

Three-dimensional surfaces of the density, molar volume and excess volume are shown in Fig. 4. The density surface has a complex shape and its approximation is the least accurate among the three DCMIX systems. The average absolute deviation between measurements and correlation is in this case $0.31 \%$. Again, the molar volume surface is flat, while the excess molar volume exhibits a sharp composition dependence and its value reaches $4 \%$, indicating that the ternary mixture water - ethanol - TEG is volumetrically non-ideal. 

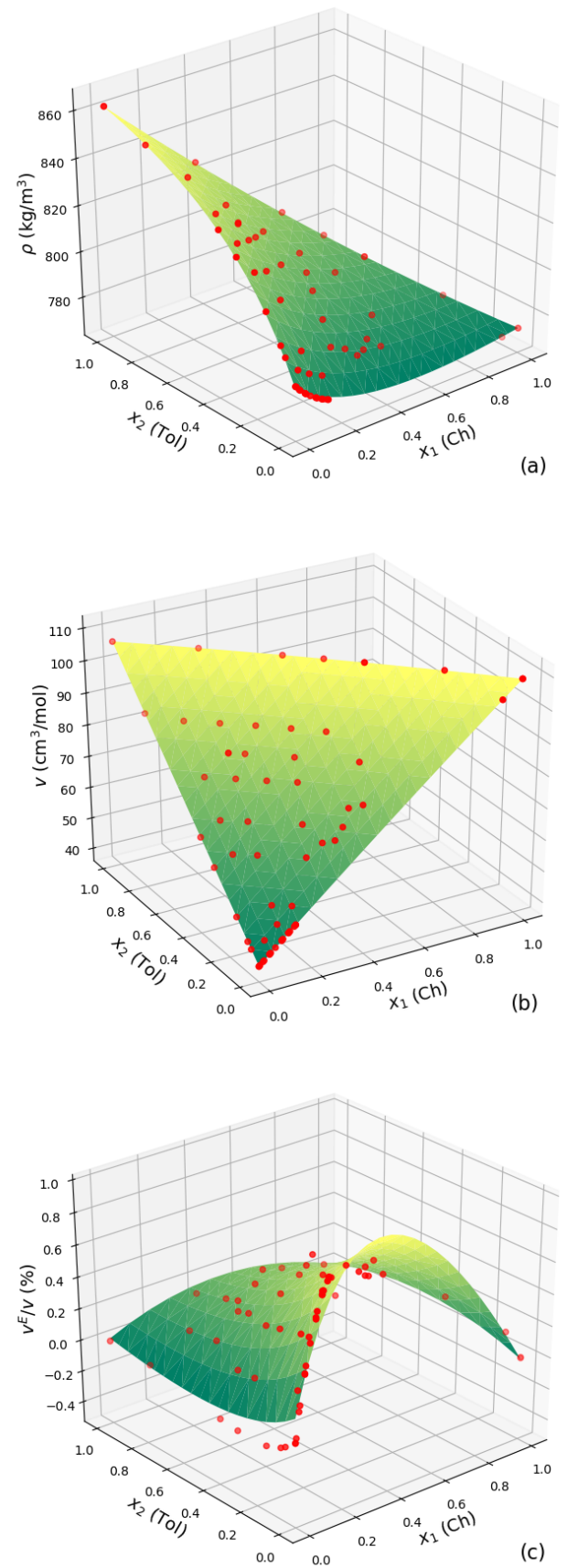

Figure 3: Ternary mixture cyclohexane (1) - toluene (2) - methanol (3): (a) specific density $\rho$; (b) molar volume $v ;$ (c) relative molar excess volume $v^{\mathrm{E}} / v$. 

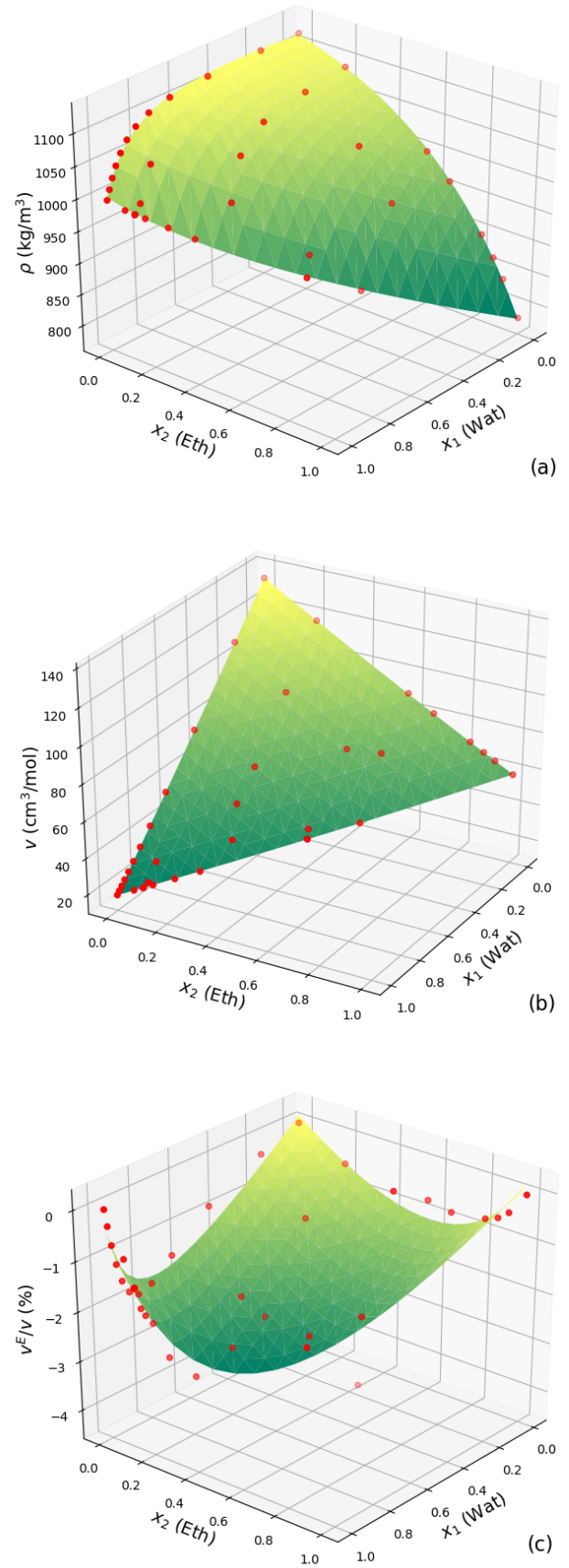

Figure 4: Ternary mixture water (1) - ethanol (2) - TEG (3) : (a) specific density $\rho$; (b) molar volume $v$; (c) relative molar excess volume $v^{\mathrm{E}} / v$. 
Table 1: Physical properties of the pure components studied during the DCMIX mission.

\begin{tabular}{llll}
\hline \hline DCMIX1 & $\mathrm{THN}$ & $\mathrm{IBB}$ & $\mathrm{nC}_{12}$ \\
\hline Chemical formula & $\mathrm{C}_{10} \mathrm{H}_{12}$ & $\mathrm{C}_{10} \mathrm{H}_{14}$ & $\mathrm{C}_{12} \mathrm{H}_{26}$ \\
Density, $\mathrm{kg} / \mathrm{m}^{3}$ & 965 & 849 & 746 \\
Molar mass, $\mathrm{g} / \mathrm{mol}$ & 132.2 & 134.22 & 170.34 \\
Molar volume, $\mathrm{cm}^{3} / \mathrm{mol}$ & 137.02 & 158.07 & 228.48 \\
\hline \hline DCMIX2 & cyclohexane & toluene & methanol \\
\hline Chemical formula & $\mathrm{C}_{6} \mathrm{H}_{12}$ & $\mathrm{C}_{7} \mathrm{H}_{8}$ & $\mathrm{CH}_{3} \mathrm{OH}$ \\
Density, $\mathrm{kg} / \mathrm{m}^{3}$ & 780 & 870 & 790 \\
Molar mass, $\mathrm{g} / \mathrm{mol}^{3}$ & 84.16 & 92.14 & 32.04 \\
Molar volume, cm $/ \mathrm{mol}$ & 108.76 & 107.05 & 40.75 \\
\hline \hline DCMIX3 & water & ethanol & $\mathrm{TEG}$ \\
\hline Chemical formula & $\mathrm{H}_{2} \mathrm{O}$ & $\mathrm{C}_{2} \mathrm{H}_{6} \mathrm{O}$ & $\mathrm{C}_{6} \mathrm{H}_{14} \mathrm{O}_{4}$ \\
Density, $\mathrm{kg} / \mathrm{m}^{3}$ & 997 & 785 & 1119 \\
Molar mass, $\mathrm{g} / \mathrm{mol}$ & 18.02 & 46.07 & 150.17 \\
Molar volume, $\mathrm{cm}^{3} / \mathrm{mol}$ & 18.08 & 58.69 & 134.2 \\
\hline \hline
\end{tabular}

\section{Results}

\subsection{Hydrocarbon mixture DCMIX1}

Relevant pure component properties of the ternary hydrocarbon mixture tetrahydronaphthalene $(\mathrm{THN})$ - isobutylbenzene $(\mathrm{IBB})$ - dodecane $\left(\mathrm{nC}_{12}\right)$ are listed in Table 1 . It can be seen that the maximum difference between the pure fluid molar volumes does not exceed $40 \%$. Because this ternary mixture is volumetrically ideal, pure fluid molar volumes can be adopted for the Fick diffusion coefficient matrix transformation. The list of 20 mixture compositions at which diffusion coefficients were measured with the Taylor dispersion technique in the volume reference frame $[12,25]$ are given in the supplementary material.

The results of the experimental data transformation to the mass and molar reference frames with Eqs. (9) and (10) are shown in Fig. 5 and listed in Table 1 of the supplementary material. In this figure, the state point numbers are plotted on the horizontal axis, where all coefficients are sorted individually in each panel in ascending order of coefficients $D_{i j}^{V}$. The main diffusion coefficient $D_{11}^{V}$ varies from 6.5 to $12.7 \cdot 10^{-10} \mathrm{~m} / \mathrm{s}^{2}$, while the variation of the other main diffusion coefficient $D_{22}^{V}$ is smaller, i.e. from 5.0 to $9.1 \cdot 10^{-10} \mathrm{~m} / \mathrm{s}^{2}$.

The prime interest of this study was the comparison of diffusion coefficients in different reference frames. In general, it was found that the diffusion coefficient values do not depend much on the reference frame. A close inspection reveals that the coefficients in the molar reference frame $D_{i j}^{M}$ show the largest digression. For example, the difference of the main diffusion coefficients in the volume and molar reference frames is noticeable and can reach $10 \%$ for $D_{11}$ and $20 \%$ for $D_{22}$.

An important conclusion of this comparison is the similarity of the main coefficients in the volume and mass reference frames, i.e. $D_{i i}^{V} \approx D_{i i}^{m}$. Furthermore, the difference between cross diffusion coefficients in these reference frames is also small. 

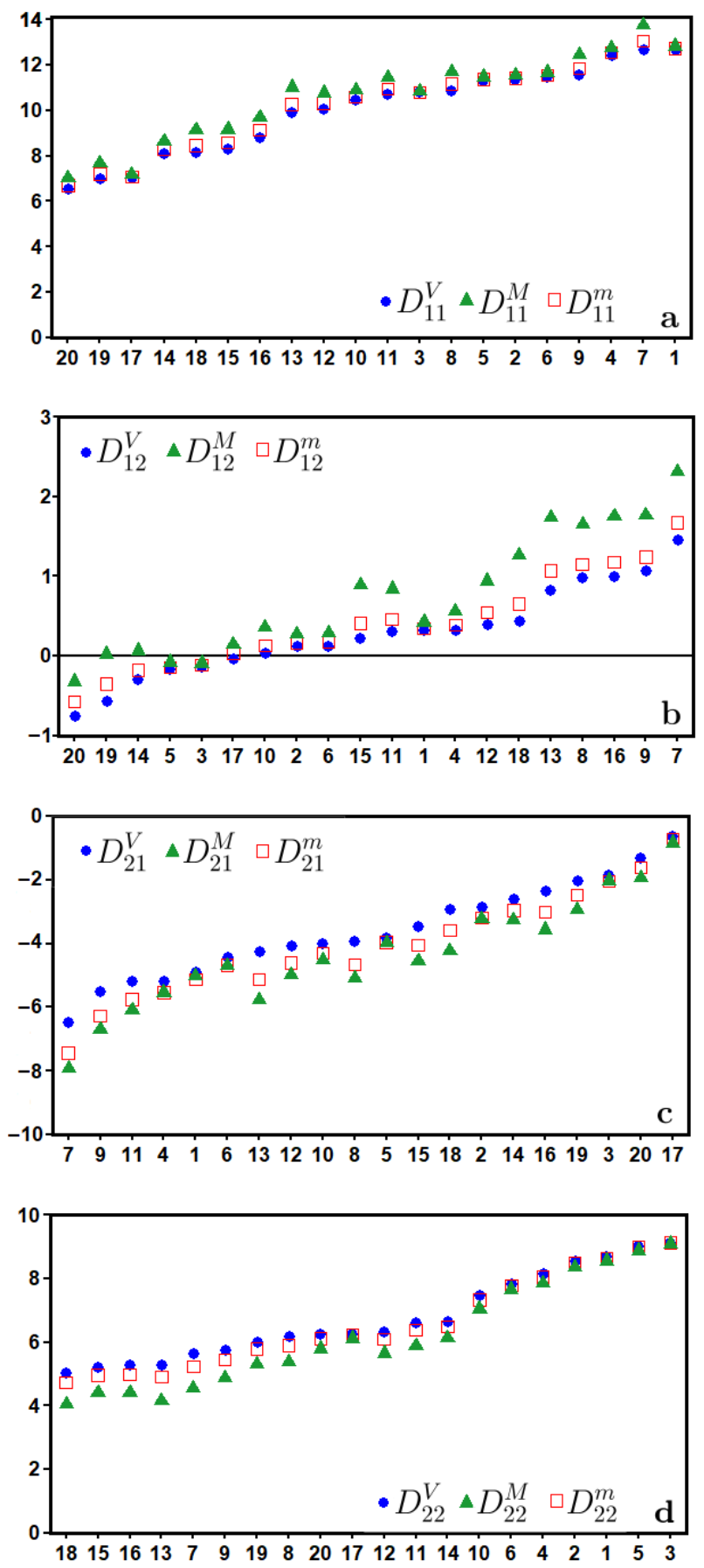

Figure 5: Fick diffusion coefficients $D_{i j} / 10^{-10} \mathrm{~m}^{2} / \mathrm{s}$ of THN (1) - IBB (2) $-\mathrm{nC}_{12}$ (3) in different reference frames. The values are sorted in ascending order of the individual coefficient in the volume reference frame. 


\subsection{Demixing mixture DCMIX2}

The mixture cyclohexane - toluene - methanol exhibits liquid-liquid phase seperation. In particular for this mixture type, having a plait point, there are ongoing discussions in the literature about the existence of negative main diffusion coefficients [5,19,23]. Our experience in measuring diffusion coefficients of liquids with a demixing zone [37] showed that the experiments are very delicate and require maintaining the specified temperature not only in the cell, but also throughout all cabling, tubes, syringes and interferometer. In particular, approaching the binodal, any unwanted small temperature variation can lead to phase separation, having a strong influence on the magnitude of the coefficients. In addition, to make definitive conclusions about the sign of the main diffusion coefficients, it is important to carry out measurements with a significant coverage of the composition space to control data evolution along composition paths. The diffusion coefficients of this mixture were measured at 57 compositions in Refs. $[7,8]$ as listed in the supplementary material. The physical properties of the pure components are given in Table 1 and the mixture density was measured in the present study. It was found that the excess volume is small, i.e. $<1 \%$, so that the transformation from volume to mass and molar reference frames was carried out with pure fluid molar volumes. The results are presented in Fig. 6 and numerical values of all coefficients are listed in supplementary material.

Surprisingly, even for this mixture with liquid-liquid phase separation not only the main diffusion coefficients, but also the cross diffusion coefficients are almost identical in the volume and mass reference frames. The diffusion coefficients in the molar reference frame differ, but the digression is not very large. It is worth noting that $D_{22}^{M}$ displays the most pronounced outliers at compositions closest to the demixing zone, where the experimental uncertainty of the cross diffusion coefficients is significant $[7,8]$.

\subsection{Associating mixture DCMIX3}

The physical properties of the pure components of water - ethanol - triethylene glycol are listed in Table 1, where it can be seen that the molar masses and molar volumes vary particularly between water and TEG by more than a factor of 7 or 8 , respectively. The partial molar volumes of this mixture are listed in Table 2 and also differ significantly from their pure fluid counterparts. Diffusion coefficients were measured in Ref. [24] at 21 compositions with the Taylor dispersion technique along several composition paths as listed in Table 2. Diffusion coefficients transformed to mass and molar reference frames are presented in Fig. 7 and listed in the supplementary material.

The variation of the main diffusion coefficients is not very sensitive to mixture composition, e.g. $D_{11}^{V}$ varies from 3 to $6.5 \cdot 10^{-10} \mathrm{~m}^{2} / \mathrm{s}$ and $D_{22}^{V}$ from 1.3 to $5.9 \cdot 10^{-10} \mathrm{~m}^{2} / \mathrm{s}$. It should be noted that one of the cross diffusion coefficients, i.e. $D_{12}^{V}$, exhibits a much larger variation, i.e. from -4.0 to $4.4 \cdot 10^{-10} \mathrm{~m}^{2} / \mathrm{s}$.

For this mixture containing three associating species, the results show a large difference between the coefficients in different reference frames. Again, the results confirm the similarity of the main diffusion coefficients in volume and mass reference frames $D_{i i}^{V} \approx D_{i i}^{m}$, but differences are notable for the cross diffusion coefficients. However, the situation is drastically different for the coefficients in the molar reference frame because the maximum discrepancy between $D_{11}^{V}$ and $D_{11}^{M}$ reaches $85 \%$. The inequality of the other coefficients between the reference frames is also significant, especially for the cross coefficient $D_{21}^{M}$, which is up to 8 times higher than $D_{21}^{V}$ for some compositions. The tendency of large differences between measured and transformed values culminates in negative main diffusion coefficients in the molar reference frame $D_{22}^{M}$, which 

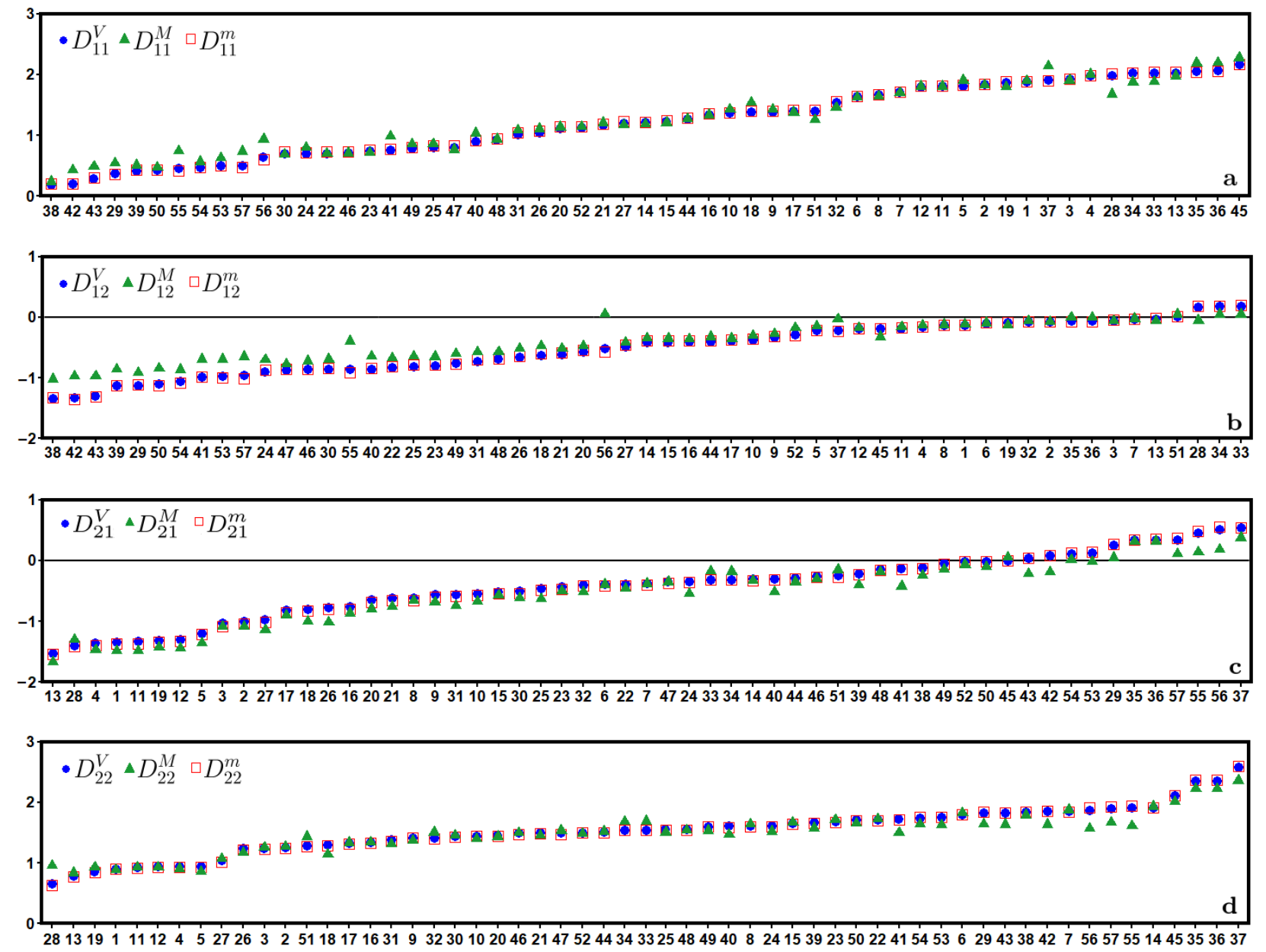

Figure 6: Fick diffusion coefficients $D_{i j} / 10^{-10} \mathrm{~m}^{2} / \mathrm{s}$ of chyclohexane (1) - toluene (2) - ethanol (3) in different reference frames. The values are sorted in ascending order of the individual coefficient in the volume reference frame. 

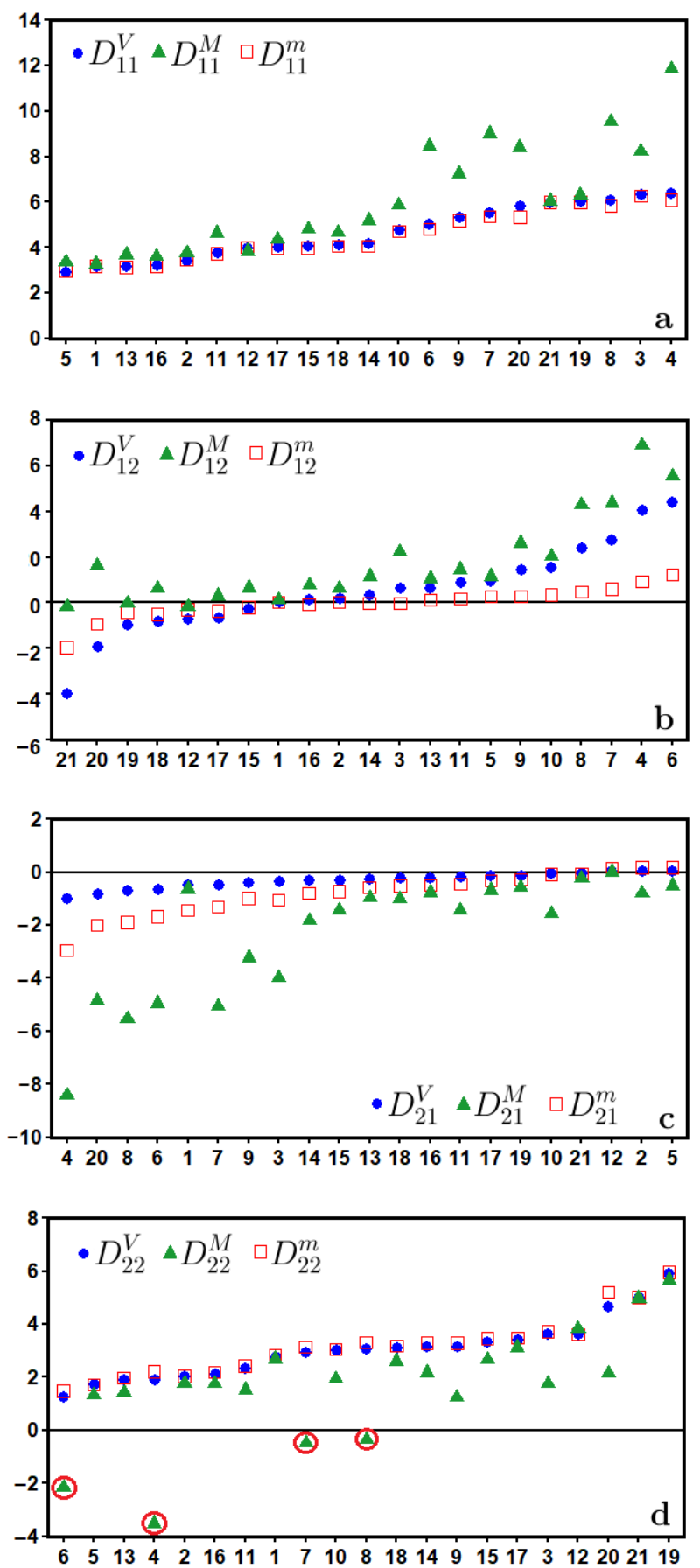

Figure 7: Fick diffusion coefficients $D_{i j} / 10^{-10} \mathrm{~m}^{2} / \mathrm{s}$ of water (1) - ethanol (2) - TEG (3) in different reference frames. The values are sorted in ascending order of the individual coefficient in the volume reference frame. 
Table 2: Ternary mixture water (1) - ethanol (2) - triethylene glycol (3): specific density $\rho / \mathrm{kg} / \mathrm{m}^{3}$, partial molar volumes $v_{i} / \mathrm{cm}^{3} / \mathrm{mol}$, molar volume $v / \mathrm{cm}^{3} / \mathrm{mol}$ and experimental [24] Fick diffusion coefficients $D_{i j}^{V} / 10^{-10} / \mathrm{m}^{2} / \mathrm{s}$.

\begin{tabular}{cccccccccccc}
\hline \hline$\#$ & $x_{1}$ & $x_{2}$ & $\rho$ & $v_{1}$ & $v_{2}$ & $v_{3}$ & $v$ & $D_{11}^{V}$ & $D_{12}^{V}$ & $D_{21}^{V}$ & $D_{22}^{V}$ \\
\hline 1 & 0.12 & 0.59 & 978.30 & 15.22 & 58.17 & 132.36 & 74.53 & 3.18 & 0.04 & -0.48 & 2.79 \\
2 & 0.35 & 0.41 & 1003.19 & 16.51 & 57.52 & 132.44 & 61.15 & 3.44 & 0.21 & 0.06 & 2.05 \\
3 & 0.47 & 0.52 & 866.59 & 16.68 & 57.73 & 131.12 & 39.17 & 6.37 & 0.62 & -0.35 & 3.62 \\
4 & 0.50 & 0.47 & 895.80 & 16.88 & 57.53 & 131.26 & 39.42 & 6.41 & 4.07 & -0.99 & 1.91 \\
5 & 0.57 & 0.22 & 1033.38 & 17.38 & 56.43 & 132.21 & 50.08 & 2.94 & 0.94 & 0.06 & 1.72 \\
6 & 0.57 & 0.31 & 975.82 & 17.31 & 56.83 & 131.77 & 43.30 & 5.02 & 4.41 & -0.63 & 1.29 \\
7 & 0.57 & 0.42 & 885.42 & 17.14 & 57.22 & 131.13 & 35.11 & 5.56 & 2.74 & -0.46 & 2.95 \\
8 & 0.58 & 0.39 & 900.05 & 17.22 & 57.10 & 131.25 & 36.19 & 6.10 & 2.43 & -0.66 & 3.07 \\
9 & 0.61 & 0.34 & 930.46 & 17.63 & 56.89 & 131.35 & 36.49 & 5.33 & 1.45 & -0.37 & 3.16 \\
10 & 0.66 & 0.26 & 974.20 & 17.56 & 56.42 & 131.45 & 36.77 & 4.76 & 1.55 & -0.04 & 3.03 \\
11 & 0.68 & 0.22 & 995.89 & 17.63 & 56.20 & 131.51 & 37.50 & 3.80 & 0.91 & -0.18 & 2.36 \\
12 & 0.75 & 0.23 & 937.27 & 17.72 & 56.04 & 130.97 & 28.80 & 3.99 & -0.70 & 0.04 & 3.65 \\
13 & 0.77 & 0.06 & 1067.19 & 17.81 & 55.16 & 130.60 & 39.40 & 3.19 & 0.64 & -0.23 & 1.90 \\
14 & 0.78 & 0.18 & 968.39 & 17.81 & 55.73 & 131.00 & 29.16 & 4.20 & 0.34 & -0.31 & 3.15 \\
15 & 0.81 & 0.13 & 1000.95 & 17.87 & 55.40 & 131.02 & 29.54 & 4.11 & -0.26 & -0.29 & 3.35 \\
16 & 0.82 & 0.03 & 1076.84 & 17.88 & 54.85 & 131.36 & 36.01 & 3.24 & 0.14 & -0.20 & 2.13 \\
17 & 0.85 & 0.07 & 1034.91 & 17.92 & 54.97 & 130.98 & 29.56 & 4.04 & -0.64 & -0.13 & 3.43 \\
18 & 0.88 & 0.02 & 1059.48 & 17.95 & 54.60 & 130.95 & 29.98 & 4.14 & -0.81 & -0.22 & 3.10 \\
19 & 0.89 & 0.10 & 965.69 & 17.95 & 54.97 & 130.53 & 22.78 & 6.05 & -0.97 & -0.11 & 5.90 \\
20 & 0.91 & 0.07 & 987.37 & 17.97 & 54.75 & 130.51 & 22.79 & 5.84 & -1.90 & -0.83 & 4.69 \\
21 & 0.95 & 0.02 & 1019.48 & 17.99 & 54.30 & 130.40 & 22.09 & 5.99 & -3.98 & -0.02 & 5.01 \\
\hline \hline
\end{tabular}

were observed for four mixture compositions (\#4,\#6, \#7, \#8 in Fig. 7 and Table 2).

It is interesting to uncover the reasons for potentially negative main diffusion coefficients. From a mathematical point of view, the transformation of diffusion matrices is reciprocal and provides equal matrix eigenvalues for all three reference frames. We are not aware of publications in which negative main diffusion coefficients were measured experimentally, except for several discrete measurements near the plait point $[18,19]$. Instead, they were obtained as a result of computations, e.g., due to solvent change [5,23]. The transformation of the diffusion matrix from the volume reference frame $\boldsymbol{D}^{V}$ into the matrix in the molar reference frame $\boldsymbol{D}^{M}$ is associated with partial molar volumes, which were discussed above. A detailed analysis of the partial molar volumes, their uncertainties and the use of volumetrically either ideal or non-ideal mixture approaches showed that corrections of the partial molar volumes alone cannot change the sign of $D_{22}^{M}$ for those four mixture compositions. Thus, partial molar volumes cannot have such an impact on computations in case of the present mixture.

Next, it was turned to the analysis of coefficient $D_{22}^{M}$, for which an analytical expression can be derived from Eq. (9). Introducing the notation

$$
\alpha=\frac{v_{3}}{v}, \quad \beta=\frac{v_{1}}{v_{3}}-1, \quad \gamma=\frac{v_{2}}{v_{3}}-1
$$

the following expression is obtained

$$
D_{22}^{M}=-x_{1} x_{2} \alpha \beta \gamma D_{11}^{V}+x_{2} \beta\left(1-x_{2} \alpha \gamma\right) D_{12}^{V}-\left(1+x_{2} \gamma\right) x_{1} \alpha \gamma D_{21}^{V}+\left(1-x_{2} \alpha \gamma\right)\left(1+x_{2} \gamma\right) D_{22}^{V}
$$

With Eq. (23), the impact of each coefficient $D_{i j}^{V}$ on the numerical value of $D_{22}^{M}$ can be discussed. It is known that the complexity of measuring ternary systems especially concerns the cross 
diffusion coefficients, which require high precision experimental data. Experimental values for $D_{12}^{V}$ and $D_{21}^{V}$ thus have larger uncertainties than the main coefficients $D_{11}^{V}$ and $D_{22}^{V}$. From Eq. (23), it can be seen that the prefactors of $D_{12}^{V}$ and $D_{21}^{V}$ have summands containing mole fractions in first order, i.e. $x_{1}$ and $x_{2}$. These summands are more significant than those in second order $x_{1}^{2}$ and $x_{2}^{2}$ because $0<x_{1}, x_{2}<1$. Consequently, their measurement uncertainty can provide a considerable contribution to the calculated $D_{22}^{M}$. The quantities $\alpha \beta \gamma$ and $\alpha \gamma^{2}$ are both positive, but they enter with a negative sign into the linear terms for $D_{12}^{V}$ and $D_{21}^{V}$. Thus, these negative factors indicate that the cross diffusion coefficients $D_{12}^{V}$ and $D_{21}^{V}$ not only strongly affect the calculation of $D_{22}^{M}$, but may lead to negative values for $D_{22}^{M}$. In case of the main coefficients $D_{11}^{V}$ and $D_{22}^{V}$, the impact of the summand with $D_{11}^{V}$ is too small, though the factor is always negative, and the summand with $D_{22}^{V}$ is always positive, which cannot lead to a negative value of coefficient $D_{22}^{M}$.

As discussed, a small alteration of measured cross diffusion coefficients $D_{12}^{V}$ and $D_{21}^{V}$ in the range of their uncertainties can change the sign of $D_{22}^{M}$. For compositions $\# 7$ and $\# 8$ (Fig. 7 and Table 2), it is sufficient to analyze coefficient $D_{21}^{V}$, where experimental values vary around zero. Thus, changing the sign of $D_{21}^{V}$ for these two values to positive, being within the assigned experimental uncertainties, positive values for coefficient $D_{22}^{M}$ are obtained. It can thus be assumed that the negative sign of $D_{22}^{M}$ at compositions \#4 and \#6 (Table 2) is related to the large experimental uncertainties of the cross diffusion coefficients, but more detailed analyses are needed.

Coefficient $D_{22}^{M}$ as a function of mass fractions is shown in Fig. 8a. Four values, which were originally found to be negative, are encircled. Fig. 8b shows the same distribution with corrected (now positive) values of $D_{22}^{M}$ for compositions $\# 7$ and $\# 8$ (Table 2), but $D_{22}^{M}$ at compositions \#4 and \#6 remains negative (encircled).

Let us consider the vicinity of compositions \#4 and \#6 in the Gibbs triangle in Fig. 8b, where only the data at these two compositions gives rise to composition range with negative values. It is known from the experimental report [34] that the optical contrast factor is zero in the binary limit $w_{1}=0.22 \mathrm{~g} / \mathrm{g}, w_{2}=0.78 \mathrm{~g} / \mathrm{g}$ and that it exhibits very small values for compositions in the area delineated in the Gibbs triangle. Thus, optical measurements of diffusion coefficients for compositions near the line of ethanol mass fraction $w_{2} \approx 0.8 \mathrm{~g} / \mathrm{g}$ were challenging and the cross diffusion coefficients $D_{12}^{V}$ and $D_{21}^{V}$ were obtained with low accuracy. Therefore, we removed compositions \#4 and \#6 from the subsequent analysis and plotted the distribution of $D_{12}^{V}, D_{21}^{V}$ (Fig. 9) and $D_{22}^{M}$ (Fig. 10a). From these figures, it can be seen which values for $D_{12}^{V}, D_{21}^{V}$ and $D_{22}^{M}$ are expected in the composition range of \#4 and \#6. Accordingly, corrected cross diffusion coefficients $D_{12}^{V}$ and $D_{21}^{V}$ were calculated at these compositions with the parametrization depicted in Fig. 9.

Based on these corrected values, the matrix transformation from the volume to the molar reference frame yields throughout positive main diffusion coefficients $D_{22}^{M}$, cf. Fig. $10 \mathrm{~b}$ and Table 3. The dark blue area in the figure contains compositions \#4 and \#6 after corrections, which match well with the adjacent data. The difference between diffusion coefficients in the three reference frames for the mixture water - ethanol - TEG in the composition region under consideration is still significant and can partly be explained by an insufficient number of experimental data points. To conclude, negative main diffusion coefficients in the molar reference frame appear as a result of the inaccurate measurement of cross diffusion coefficients, which is strongly amplified by the transformation between reference frames. 

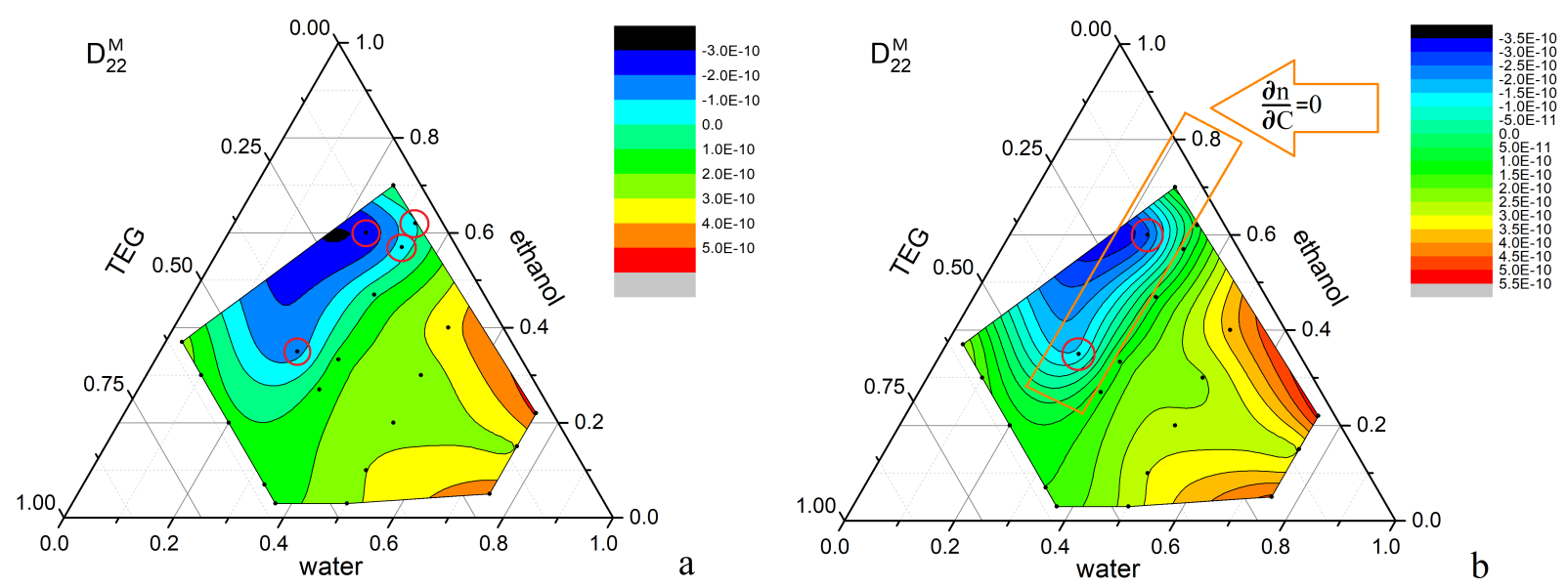

Figure 8: Distribution of the main Fick diffusion coefficient $D_{22}^{M}$ of water (1) - ethanol (2) - TEG (3) over mass fractions: (a) without correction; (b) with correction for two compositions \#7 and \#8.

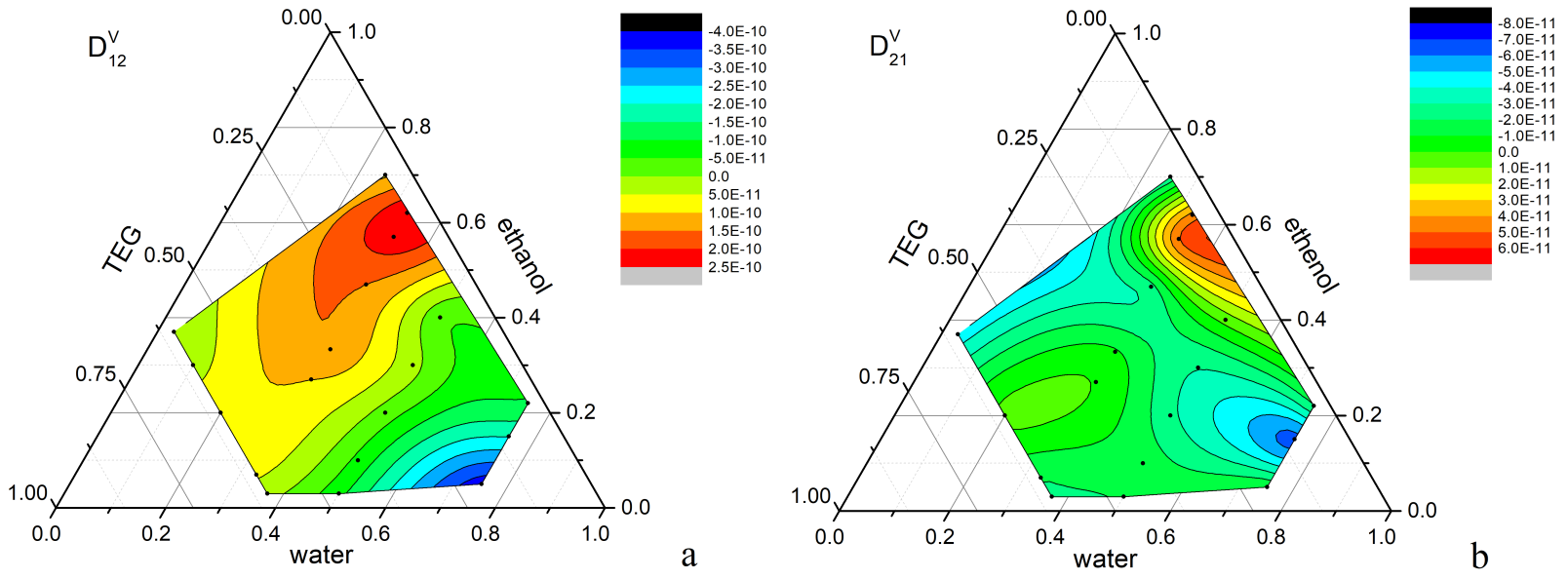

Figure 9: Distribution of the cross Fick diffusion coefficients $D_{12}^{V}$ (a) and $D_{21}^{V}$ (b) of water (1) - ethanol (2) TEG (3) over mass fractions (omitting two compositions).
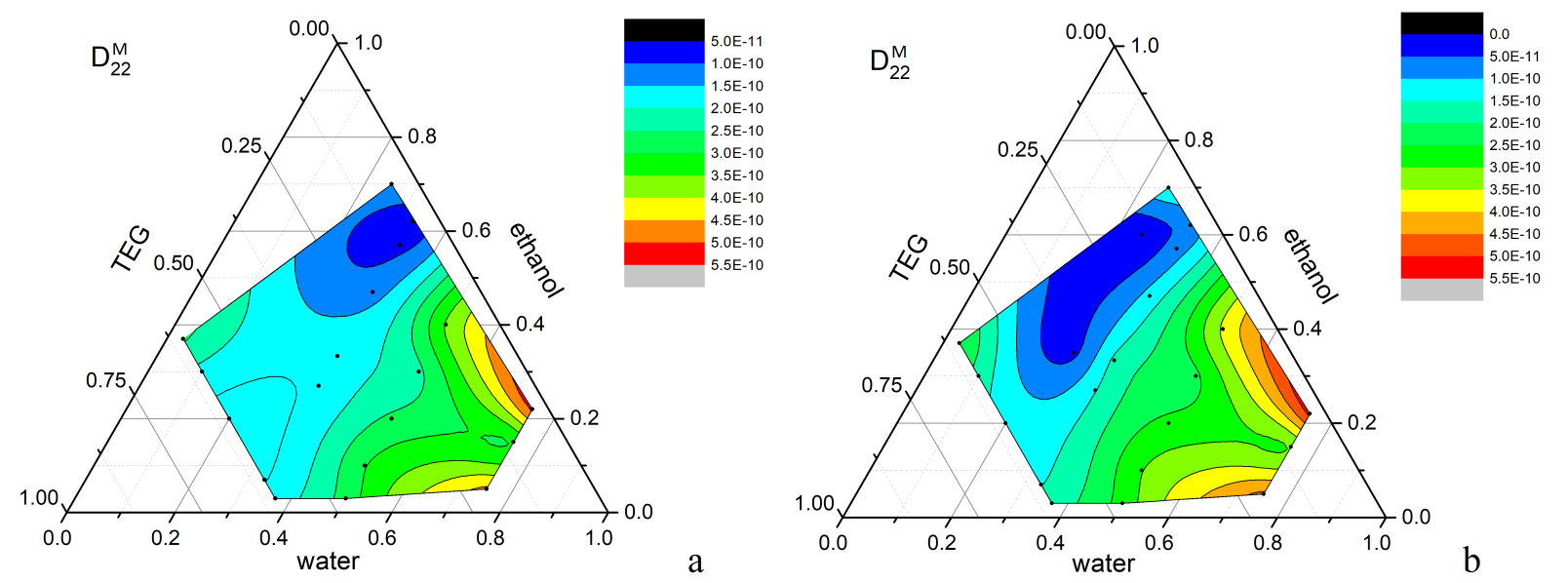

Figure 10: Distribution of the main Fick diffusion coefficient $D_{22}^{M}$ of water (1) - ethanol (2) - TEG (3) over mass fractions: (a) omitting two compositions; (b) with coefficient corrections. 

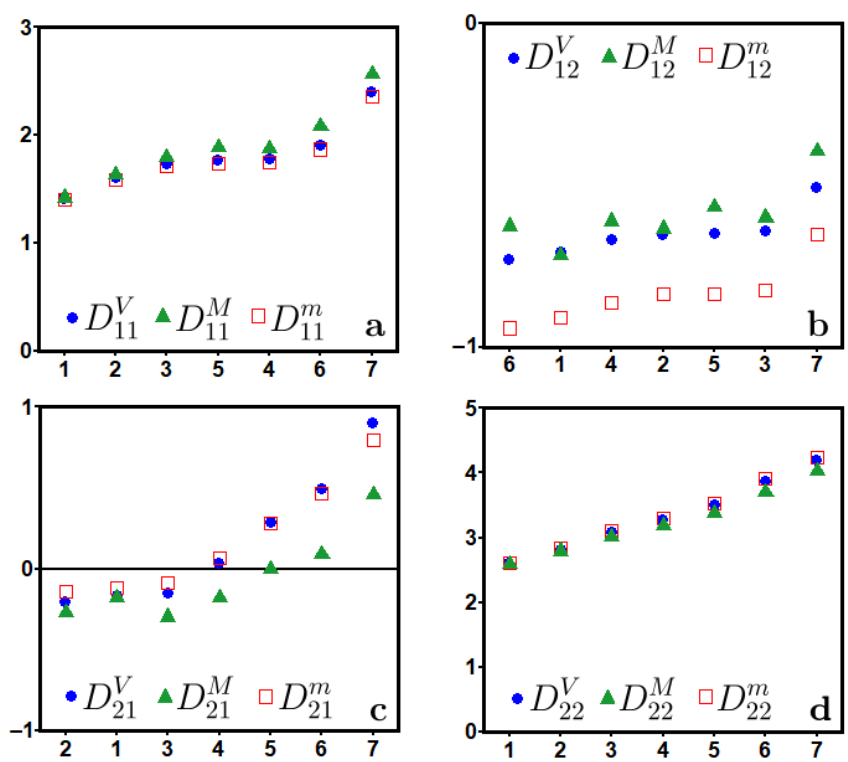

Figure 11: Fick diffusion coefficients $D_{i j} / 10^{-10} \mathrm{~m}^{2} / \mathrm{s}$ of benzene (1) - acetone (2)- ethanol (3) in different reference frames. The values are sorted in ascending order of the individual coefficient in the volume reference frame.

Table 3: Correction of original data for cross diffusion coefficients $D_{i j}^{V} / 10^{-10} \mathrm{~m}^{2} / \mathrm{s}$ of water $(1)$ - ethanol (2) TEG (3) and its impact on $D_{22}^{M} / 10^{-10} \mathrm{~m}^{2} / \mathrm{s}$ in the molar reference frame.

\begin{tabular}{cccccccccc}
\hline \hline & \multicolumn{1}{c}{ original } & \multicolumn{3}{c}{ corrected } \\
\hline$\#$ & $x_{1}$ & $x_{2}$ & $D_{12}^{V}$ & $D_{21}^{V}$ & $D_{22}^{M}$ & $D_{12}^{V}$ & $D_{21}^{V}$ & $D_{22}^{M}$ \\
\hline 4 & 0.50 & 0.47 & 4.07 & -0.99 & -3.53 & 0.50 & 0.30 & 0.033 \\
6 & 0.57 & 0.31 & 4.41 & -0.63 & -2.16 & 0.5 & 0.01 & 0.028 \\
7 & 0.57 & 0.42 & 2.74 & -0.46 & -0.49 & 2.74 & 0.46 & 0.33 \\
8 & 0.58 & 0.39 & 2.43 & -0.66 & -0.36 & 2.43 & 0.66 & 0.89 \\
\hline \hline
\end{tabular}

\subsection{Other mixtures}

In addition to the three DCMIX mixtures, we considered three more mixtures for which diffusion coefficients were measured along one composition path each: benzene - acetone - methanol, benzene - acetone - ethanol, benzene - acetone - 2-propanol.

The diffusion coefficients in the first three mixtures, composed of an aromatic (benzene), a ketone (acetone) and one of the alcohols methanol, ethanol or 2-propanol were recently experimentally measured and predicted by molecular dynamics simulation at nine compositions along a single composition path with a constant benzene mole fraction $x_{1}=0.33 \mathrm{~mol} \mathrm{~mol}^{-1}[15$, 16]. The excess volume of these mixtures is small and the transformation between reference frames was made on the basis of the pure fluid molar volumes. The results show that the main diffusion coefficients are similar in all three reference frames (Fig. 11), as it was observed above for the hydrocarbon mixture. Cross diffusion coefficients exhibit only a small discrepancy between the volume and mass reference frames, while divergence is a slightly larger for the molar reference frame.

We also examined the diffusion coefficients of the non-ideal system water - methanol - 
ethanol recently measured along two composition paths [14] and the results confirm the observation that the main diffusion coefficients in the molar reference frame exhibit a larger divergence from the other frames, but they are throughout positive. It is worth noting that half a century ago diffusion coefficients were measured for the moderately non-ideal system acetone - benzene - carbon tetrachloride with the diaphragm cell method, relying on a different mathematical approach [38]. For a large number of compositions, all main diffusion coefficients were reported to be positive.

\section{Conclusion}

The dependence of Fick diffusion coefficients of ternary mixtures upon transformation between different reference frames was analyzed. For this purpose, three mixture types were considered, for which diffusion coefficients were measured over a large compositions range: (1) the hydrocarbon mixture tetralin - isobutylbenzene - dodecane; (2) a mixture with liquidliquid phase separation cyclohexane - toluene - methanol and (3) a mixture containing three associating components water - ethanol - triethylene glycol. A quick examination was done for three more mixtures composed of benzene, acetone and one alcohol (methanol, ethanol or 2-propanol) for which diffusion coefficients were measured along one composition path each. Similarly, water - methanol - ethanol was explored along two composition paths. The considered experimental Fick diffusion data were obtained by several research groups throughout with the Taylor dispersion technique.

It was focused on the transformation of Fick diffusion coefficients from the volume to the molar and mass reference frames. To use appropriate partial molar volumes for this task, the mixture density was measured in the present work. An analysis of the excess molar volume showed that its values are negligibly small for all considered mixtures, except for water - ethanol - triethylene glycol. For this case, partial molar volumes were derived from experimental density data of this volumetrically non-ideal mixture, while pure fluid molar volumes were used for the remaining ones.

This study was devoted to the existence of negative main Fick diffusion coefficients and the disclosure of their possible cause. This subject is under discussion for several decades, in particular for ternary mixtures with a plait point. Only recently, more extensive data sets appeared for ternary systems, where diffusion coefficients were measured for a large number of compositions.

One of the key findings is that the main diffusion coefficients are similar in volume and mass reference frames for all considered mixtures, even for water - ethanol - triethylene glycol. For the latter mixture, the dependence on the reference frame is greater than for the other ones. Furthermore, mixtures with a small molar excess volume $(<1 \%)$ exhibit cross diffusion coefficients with similar tendencies in all three reference frames.

However, the evolution of diffusion coefficients in the molar reference frame is quite different. The main and especially the cross diffusion coefficients deviate from their associates in the other two reference frames, and the discrepancy grows with an increase of the excess volume and in the region where it varies strongly. In addition, the diffusion coefficient matrix transformed to the molar reference frame $\boldsymbol{D}^{M}$ exhibits a strong dependence on the values of the cross diffusion coefficients $D_{i j}^{V}$.

Negative values for the main diffusion coefficient in the molar reference frame $D_{22}^{M}$ were found for the mixture water - ethanol - triethylene glycol at four compositions. Deeper analyses have shown that they occur in composition ranges with poor optical properties, where cross dif- 
fusion coefficients were measured with large uncertainties. Their impact during transformation is so profound that it results in a negative main diffusion coefficient $D_{22}^{M}$. Analyzing the cause and correcting the cross diffusion coefficients $D_{i j}^{V}$, it was evidenced that the main elements are throughout positive in all reference frames. Thus, another key finding is that the appearance of negative diffusion coefficients $D_{22}^{M}$ is associated with a large uncertainty in measured cross diffusion coefficients which is strongly amplified upon transformation between reference frames.

\section{Acknowledgments}

SK was funded by Russian Foundation for Basic Research, Government of Krasnoyarsk Territory, Krasnoyarsk Regional Fund of Science to the research project N 18-41-243005. AM and VS were supported by the Belgian Federal Science Policy Office (PRODEX program of ESA). TJ and JV acknowledge financial support from Deutsche Forschungsgemeinschaft under the grant VR 6/11.

\section{References}

[1] Taylor, R., and Krishna, R., Multicomponent mass transfer, Wiley Series in Chemical Engineering, 2nd edition, 1993.

[2] Budroni, M. A., Lemaigre, L., De Wit, A., Rossi, F. Cross-diffusion-induced convective patterns in microemulsion systems, Phys. Chem. Chem. Phys., 17, pp. 1593-1600 (2015).

[3] Bratsun, D. A., Stepkina, O. S., Kostarev, K. G., Mizev, A. I., Mosheva, E. A. Development of Concentration-Dependent Diffusion Instability in Reactive Miscible Fluids Under Influence of Constant or Variable Inertia, Microgravity Sci. Technol., 28, pp. 575-585 (2016).

[4] Zhang, H., Annunziata, O. Effect of macromolecular polydispersity on diffusion coefficients measured by Rayleigh interferometry, J. Phys. Chem. B., 112, pp. 3633-3643 (2008).

[5] Buzatu, D., Buzatu, F. D. Paduano L., Roberto Sartorio, R. Diffusion Coefficients for the Ternary System Water + Chloroform + Acetic Acid at $25^{\circ} \mathrm{C}$, J. Solution Chem., 36, pp. 1373-1384 (2007).

[6] Chen, L., Leaist, D. G. Multicomponent Taylor Dispersion Coefficients, J. Solution Chem., 43, pp. 2224-2237 (2014).

[7] Grossmann, T., Winkelmann, J. Ternary Diffusion Coefficients of Cyclohexane + Toluene + Methanol by Taylor Dispersion Measurements at 298.15 K. Part 1. Toluene-Rich Area, J. Chem. Eng. Data, 54, pp. 405-410 (2009).

[8] Grossmann, T., Winkelmann, J. Ternary Diffusion Coefficients of Cyclohexane + Toluene + Methanol by Taylor Dispersion Measurements at 298.15 K. Part 2. Low Toluene Area Near the Binodal Curve, J. Chem. Eng. Data, 54, pp. 485-490 (2009).

[9] Larrañaga, M., Rees, D. A., Bou-Ali, M. M. Determination of the molecular diffusion coefficients in ternary mixtures by the sliding symmetric tubes technique, J. Chem. Phys., 140, 054201 (2014). 
[10] Mialdun, A., Yasnou, V., Shevtsova, V. Measurement of isothermal diffusion coefficients in ternary mixtures using counter flow diffusion cell, C. R. Mecanique, 341, pp. 462-468 (2013).

[11] Mialdun, A., Shevtsova, V. Analysis of multi-wavelength measurements of diffusive properties via dispersion dependence of optical properties, Appl. Opt., 56, pp. 572-581 (2017).

[12] Mialdun, A., Sechenyh, V., Legros, J. C., Ortiz de Zárate, J. M., Shevtsova, V. Investigation of Fickian diffusion in the ternary mixture of 1,2,3,4-tetrahydronaphthalene, isobutylbenzene, and dodecane, J. Chem. Phys., 139, 104903 (2013).

[13] Mutoru, J. W., Firoozabadi, A. Form of multicomponent Fickian diffusion coefficients matrix, J. Chem. Thermodynamics, 43, pp. 1192-1203 (2011).

[14] Guevara-Carrion, G., Gaponenko, Y., Janzen, T., Vrabec J., Shevtsova, V. Diffusion in Multicomponent Liquids: From Microscopic to Macroscopic Scales, J. Phys. Chem. B, 120, pp. 12193-12210 (2016).

[15] Janzen, T., Gaponenko, Y., Mialdun, A. Guevara-Carrion, G. and Vrabec, J., Shevtsova, $\mathrm{V}$. The effect of alcohols as the third component on diffusion in mixtures of aromatics and ketones, RSC Adv., 8(18), pp. 10017-10022 (2018)

[16] Guevara-Carrion, G., Gaponenko, Y., Mialdun, A., Janzen, T., Shevtsova, V., Vrabec J., Interplay of structure and diffusion in ternary liquid mixtures of benzene + acetone + varying alcohols, J. Chem. Phys., 149, 064504 (2018).

[17] Miller, D. G. Some comments on multicomponent diffusion: negative main term diffusion coefficients, second law constraints, solvent choices, and reference frame transformations, J. Phys. Chem., 90 pp. 1509-1519 (1986).

[18] Kirkaldy, J. S., Purdy, G. R. Diffusion in multicomponent metallic systems. X. Diffusion at and near ternary critical state, Can. J. Phys, 47, pp. 865-871 (1969).

[19] Clark, W. M., Rowley, R. L. Ternary Liquid Diffusion Coefficients Near Plait Points, Int. J. Thermophys., 6, pp. 631-642 (1985).

[20] Cussler, E. L. Multicomponent Diffusion, Elsevier, Amsterdam, 1976.

[21] Tyrell, H. J. V., Harris, K. R. Diffusion in Liquids, Butterworths, London, 1984.

[22] Chen, Q., Engström, A., Ågren, J., On negative diagonal dlements in the diffusion coefficient matrix of multicomponent systems, J. Phase Equilib. Diffus. 39(5) pp.592-596 (2018)

[23] Vitagliano, V., Sartorio, R., Scala, S., Spaduzzi, D. Diffusion in a Ternary System and the Critical Mixing Point, J. Solution Chem., 7, pp. 605-621 (1978).

[24] Legros, J. C., Gaponenko, Y., Mialdun, A., Triller, T., Hammon, A., Bauer, C., Köhler, W., Shevtsova V. Investigation of Fickian diffusion in the ternary mixtures of waterethanoltriethylene glycol and its binary pairs, Phys. Chem. Chem. Phys., 17, pp. 27713-27725 (2015).

[25] Sechenyh, V., Legros, J. C., Mialdun, A., Ortiz de Zárate, J. M., Shevtsova, V. Fickian Diffusion in Ternary Mixtures Composed by 1,2,3,4-Tetrahydronaphthalene, Isobutylbenzene, and n-Dodecane, J. Phys. Chem. B, 120, pp. 535-548 (2016). 
[26] Bou-Ali, M. M., Ahadi, A., Alonso de Mezquia, D., Galand, Q., Gebhardt, M., Khlybov, O., Köhler, W., Larrañaga, M., Legros, J. C., Lyubimova, T., Mialdun, A., Ryzhkov, I., Saghir, M. Z., Shevtsova, V., Van Vaerenbergh, S. Benchmark values for the Soret, thermodiffusion and molecular diffusion coefficients of the ternary mixture tetralin+isobutylbenzene+n-dodecane with 0.8-0.1-0.1 mass fraction, Eur. Phys. J. E, 38, $30(2015)$.

[27] Mialdun, A., Shevtsova, V. Temperature dependence of Soret and diffusion coefficients for toluene-cyclohexane mixture measured in convection-free environment, J. Chem. Phys., 143, 224902 (2015).

[28] Mialdun, A., Ryzhkov, I., Khlybov, O., Lyubimova, T., Shevtsova, V. Measurement of Soret coefficients in a ternary mixture of toluene-methanol-cyclohexane in convection-free environment, J. Chem. Phys., 148, 044506 (2018).

[29] Triller, T., Bataller, H., Bou-Ali, M. M., Braibanti, M., Croccolo, F., Ezquerro, J. M., Galand, Q., Gavaldà, Jna, Lapeira, E., Laverón-Simavilla, A., Lyubimova, T., Mialdun, A., Ortiz de Zárate, J. M., Rodríguez, J., Ruiz, X., Ryzhkov, I. I., Shevtsova, V., Van Vaerenbergh, S., Köhler, W. Thermodiffusion in Ternary Mixtures of Water/Ethanol/Triethylene Glycol: First Report on the DCMIX3-Experiments Performed on the International Space Station, Microgravity Sci. Technology, 30, pp. 295308 (2018)

[30] Lapeira, E., Gebhardt, M., Triller, T., Mialdun, A., Köhler, W., Shevtsova, V., Bou-Ali, M. M. Transport properties of the binary mixtures of the three organic liquids toluene, methanol, and cyclohexane, J. Chem. Phys., 146, 094507 (2017).

[31] Guevara-Carrion., G., Janzen, T., Munoz-Munoz, Y. M., Vrabec, J. Mutual diffusion of binary liquid mixtures containing methanol, ethanol, acetone, benzene, cyclohexane, toluene and carbon tetrachloride, J. Chem. Phys., 144, 124501 (2016).

[32] Santos, C. I. A. V., Shevtsova, V., Burrows, H. D., Ribeiro, A. C. F. Optimization of Taylor Dispersion Technique for Measurement of Mutual Diffusion in Benchmark Mixtures, Microgravity Sci. Technol., 28, pp. 459-465 (2016).

[33] Shevtsova, V., Santos, C., Sechenyh, V., Legros, J.C., Mialdun, A. Diffusion and Soret in Ternary Mixtures. Preparation of the DCMIX2 Experiment on the ISS, Microgravity Sci. Technol. 25, pp. 275-283 (2014)

[34] Königer, A., Meier, B., Köhler, W. Measurement of the Soret, diffusion, and thermal diffusion coefficients of three binary organic benchmark mixtures and of ethanol-water mixtures using a beam deflection technique, Phil. Magazine, 89, pp. 907-923 (2009).

[35] Lapeira, E., Bou-Ali, M. M., Madariaga, J. A., Santamaría, C. Thermodiffusion Coefficients of Water/Ethanol Mixtures for Low Water Mass Fractions, Microgravity Sci. Technol., 28, pp. 553-557 (2016).

[36] Eslamian, M., Jiang, C. G., Saghir M. Z. , Role of the velocity frame of reference in thermodiffusion in liquid mixtures, Philosophical Magazine, 92(6), pp. 705-726 (2012).

[37] Janzen, T., Zhang, S., Mialdun, A., Guevara-Carrion, G., Vrabec, J., He, M., Shevtsova, V. Mutual diffusion governed by kinetics and thermodynamics in the partially miscible mixture methanol + cyclohexane, Phys. Chem. Chem. Phys., 19, pp. 31856-31873 (2017). 
[38] H.T. Cullinan Jr., Toor H.L., Diffusion in the three-component liquid system acetonebenzene-Carbon Tetrachloride, J. Phys. Chem., 69, pp. 3941-3949 (1965). 


\title{
Do ternary liquid mixtures exhibit negative main Fick diffusion coefficients?
}

\author{
S. Kozlova ${ }^{1}$, A. Mialdun ${ }^{2}$, I. Ryzhkov ${ }^{1,4}$, T. Janzen $^{3}$, J. Vrabec $^{3}$ and V. \\ Shevtsova $^{2}$ \\ ${ }^{1}$ Institute of Computational Modelling SB RAS, \\ 50/44 Akademgorodok, 660036, Krasnoyarsk, Russia \\ 2 Microgravity Research Center, Université Libre de Bruxelles (ULB), \\ CP-165/62, Av. F.D. Roosevelt, 50, B-1050 Brussels, Belgium \\ Thermodynamics and Thermal Process Engineering, Technical University \\ Berlin, \\ Ernst-Reuter-Platz 1, 10587 Berlin, Germany \\ ${ }^{4}$ Siberian Federal University \\ 79 Svobodny pr., 660041, Krasnoyarsk, Russia
}

\section{Partial molar volumes of a binary mixture}

The molar volume of a binary liquid mixture at constant temperature and pressure is in general given by

$$
v=x_{1} v_{1}+x_{2} v_{2}
$$

On the other hand, the molar volume of a volumetrically ideal binary mixture is

$$
v=\frac{M}{\rho}=\frac{x_{1} M_{1}}{\rho}+\frac{x_{2} M_{2}}{\rho}=x_{1} v_{1}^{0}+x_{2} v_{2}^{0},
$$

where $M$ and $M_{i}$ are the molar mass of the mixture and its components.

In a volumetrically non-ideal mixture, the total volume is not the weighted sum of the pure component volumes $v_{i}^{0}$ and the partial molar volumes $v_{i}$ depend on mixture composition such that they can be smaller or larger than $v_{i}^{0}$ due to volumetric contraction or dilatation.

Considering Eq. (1) under isothermal-isobaric conditions leads to

$$
d v=x_{1} d v_{1}+v_{1} d x_{1}+x_{2} d v_{2}+v_{2} d x_{2} .
$$

The Gibbs-Duhem equation yields $x_{1} d v_{1}+x_{2} d v_{2}=0$ so that

$$
d v=v_{1} d x_{1}+v_{2} d x_{2} .
$$

From the closure $x_{1}+x_{2}=1$, it follows that $d x_{1}=-d x_{2}$. Substituting this into Eq. (4) yields $d v=v_{1} d x_{1}-v_{2} d x_{1}$ and

$$
\frac{d v}{d x_{1}}=v_{1}-v_{2}=v_{1}-\frac{v-x_{1} v_{1}}{x_{2}}
$$

considering Eq. (1). Finally,

$$
v_{1}=v+\left(1-x_{1}\right) \frac{d v}{d x_{1}} .
$$

Accordingly, the values of $v_{1}$ and $v_{2}$ obviously depend on mixture composition. 


\section{Examined compositions}
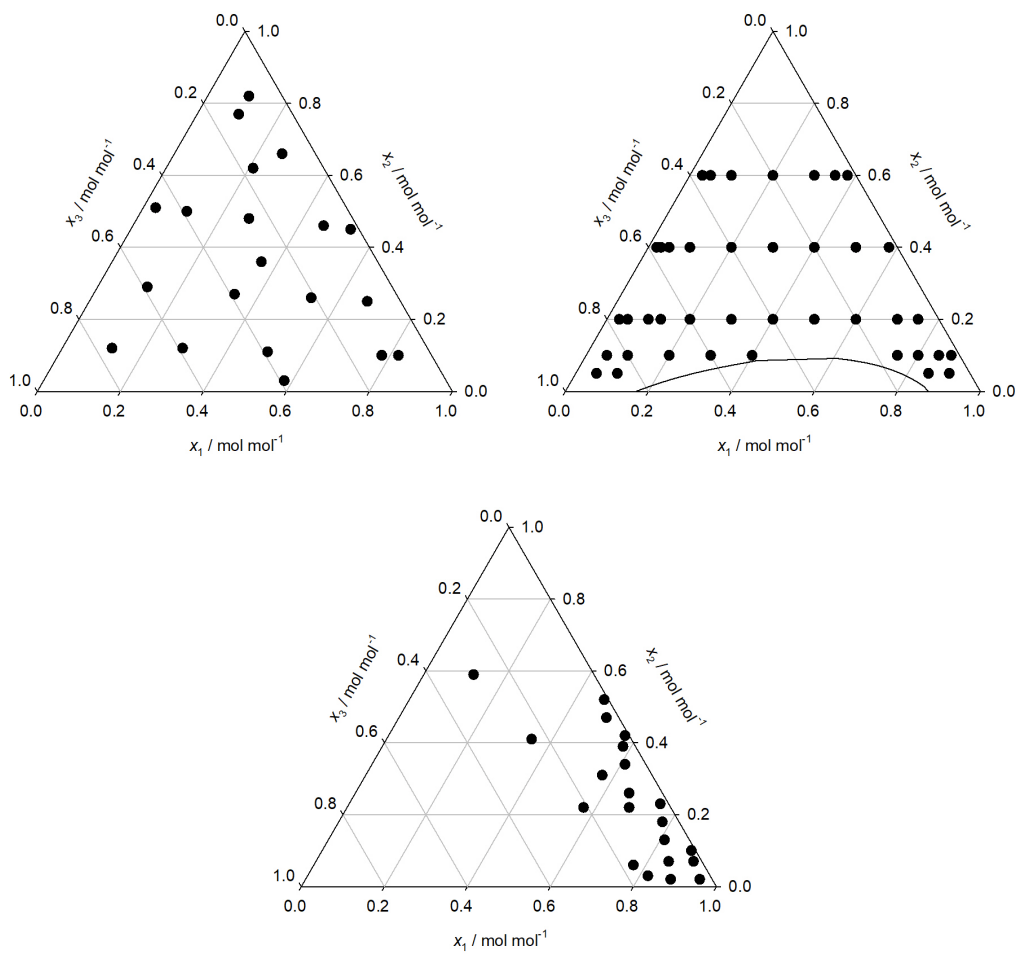

Figure 1: Compositions in terms of mole fractions at which their diffusion coefficients were measured : (a) THN - IBB $-\mathrm{nC}_{12}$; (b) cyclohexane - toluene methanol; (c) water - ethanol - TEG.

\section{Tabular data for examined mixtures}




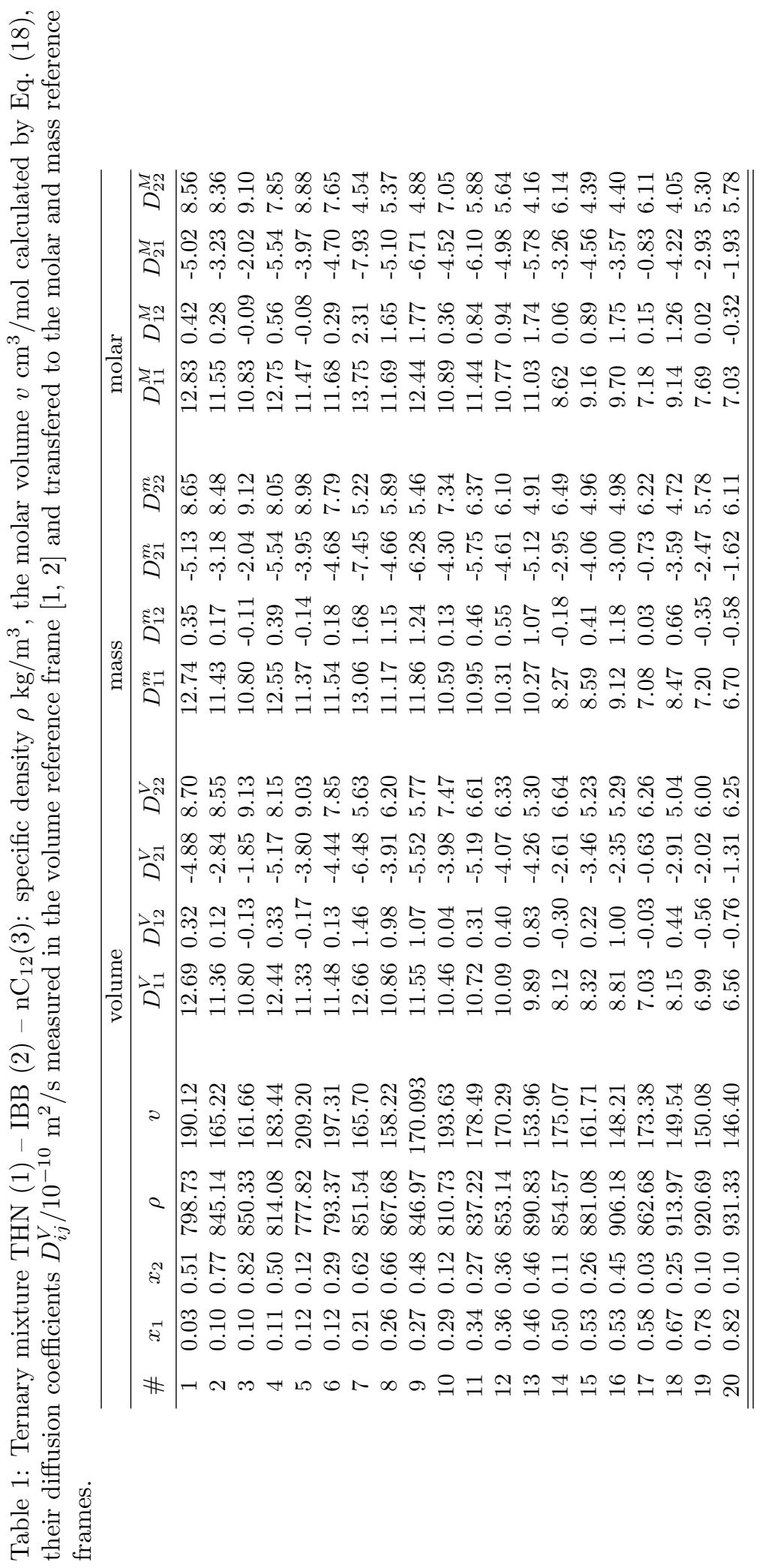




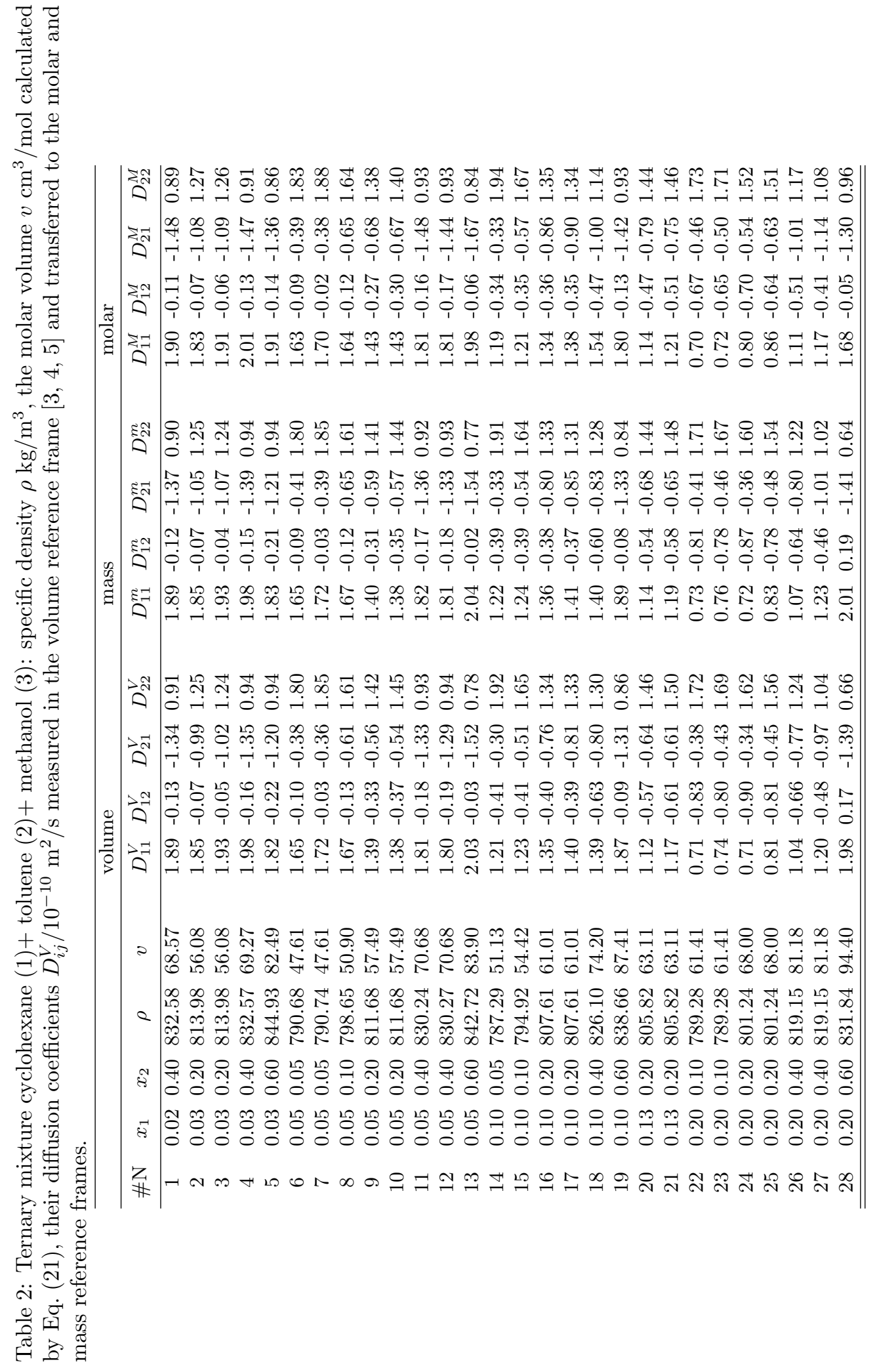




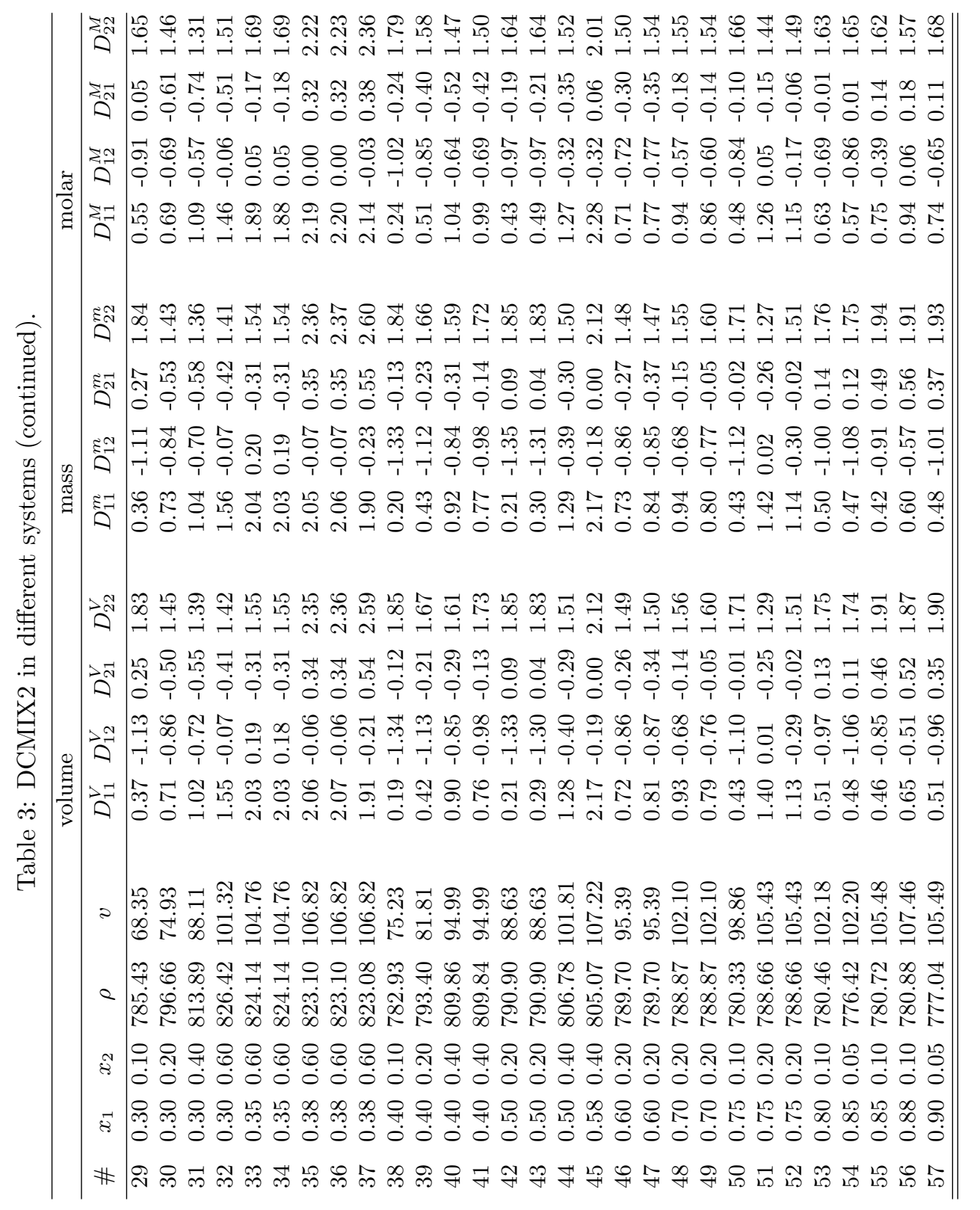




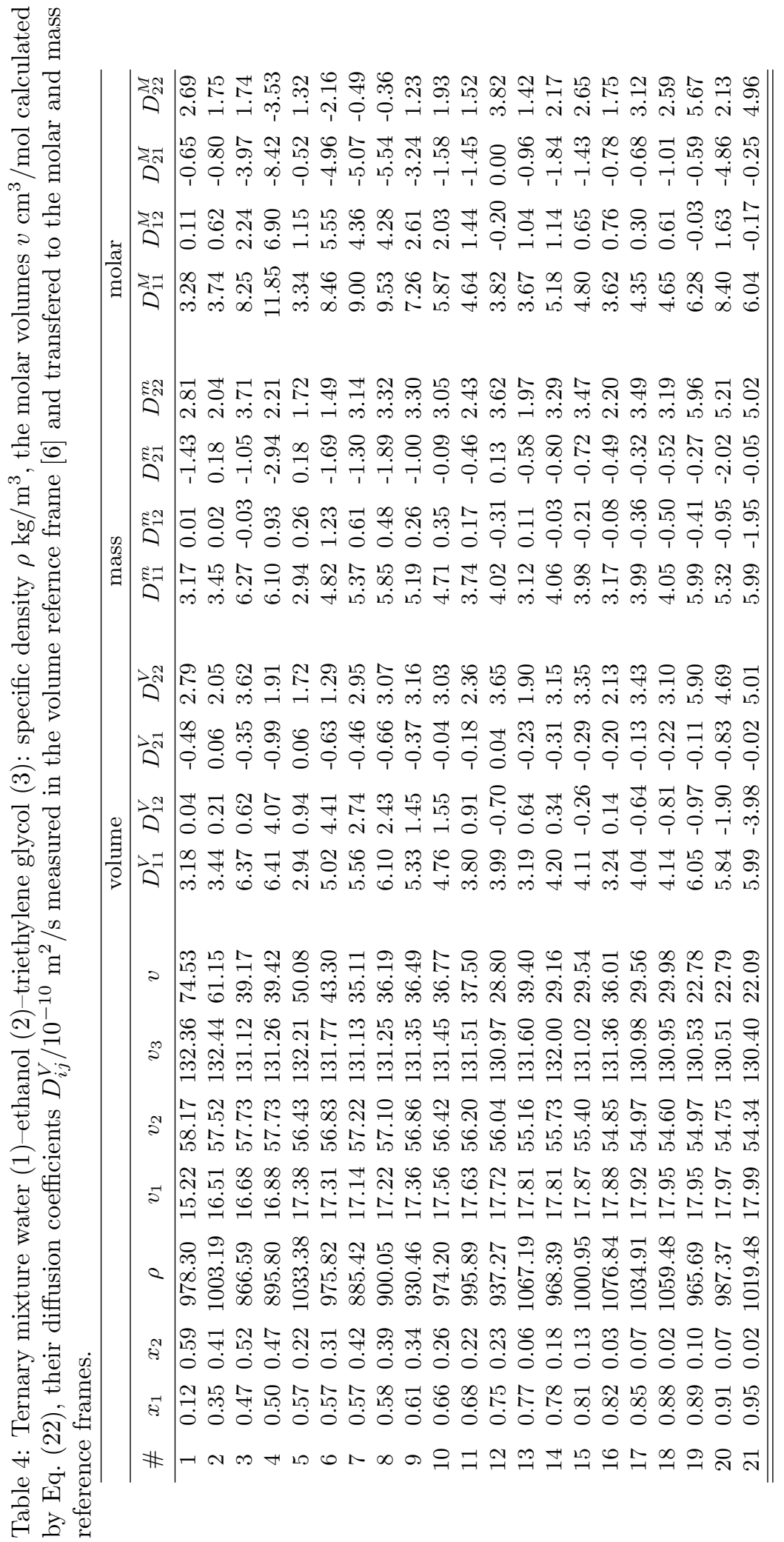




\section{Acknowledgements}

\section{References}

[1] Mialdun, A., Sechenyh, V., Legros, J. C., Ortiz de Zárate, J. M., Shevtsova, V. Investigation of Fickian diffusion in the ternary mixture of 1,2,3,4tetrahydronaphthalene, isobutylbenzene, and dodecane, J. Chem. Phys., 139, 104903 (2013).

[2] Sechenyh, V., Legros, J. C., Mialdun, A., Ortiz de Zárate, J. M., Shevtsova, V. Fickian Diffusion in Ternary Mixtures Composed by 1,2,3,4Tetrahydronaphthalene, Isobutylbenzene, and n-Dodecane, J. Phys. Chem. B, 120, pp. 535-548 (2016).

[3] Grossmann, T., Winkelmann, J. Ternary Diffusion Coefficients of Cyclohexane + Toluene + Methanol by Taylor Dispersion Measurements at 298.15 K. Part 1. Toluene-Rich Area, J. Chem. Eng. Data, 54, pp. 405-410 (2009).

[4] Grossmann, T., Winkelmann, J. Ternary Diffusion Coefficients of Cyclohexane + Toluene + Methanol by Taylor Dispersion Measurements at 298.15 K. Part 2. Low Toluene Area Near the Binodal Curve, J. Chem. Eng. Data, 54, pp. 485-490 (2009).

[5] Santos, C.I., Shevtsova, V., Ribeiro, A.C., Isothermal molecular diffusion in mixtures containing toluene, cyclohexane and methanol. Eur. Phys. J. E, 40, 40 (2017).

[6] Legros, J. C., Gaponenko, Y., Mialdun, A., Triller, T., Hammon, A., Bauer, C., Köhler, W., Shevtsova V. Investigation of Fickian diffusion in the ternary mixtures of waterethanoltriethylene glycol and its binary pairs, Phys. Chem. Chem. Phys., 17, pp. 27713-27725 (2015). 